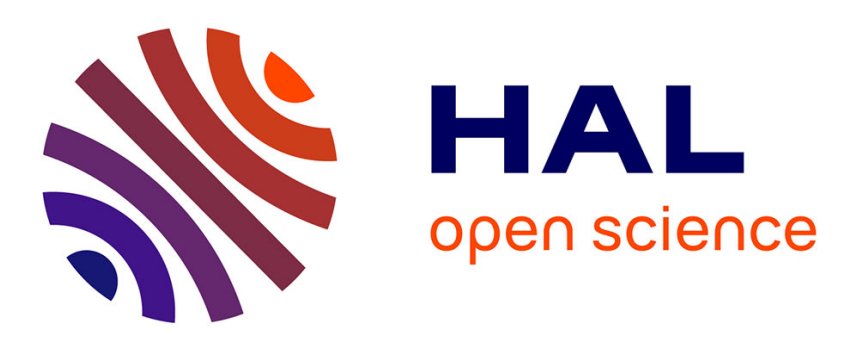

\title{
Transition to chaotic vibrations for harmonically forced perfect and imperfect circular plates
}

\author{
Cyril Touzé, Olivier Thomas, Marco Amabili
}

\section{To cite this version:}

Cyril Touzé, Olivier Thomas, Marco Amabili. Transition to chaotic vibrations for harmonically forced perfect and imperfect circular plates. International Journal of Non-Linear Mechanics, 2011, 46 (1), pp.234-246. 10.1016/j.ijnonlinmec.2010.09.004 . hal-01134807

\section{HAL Id: hal-01134807 \\ https://hal-ensta-paris.archives-ouvertes.fr/hal-01134807}

Submitted on 14 Mar 2016

HAL is a multi-disciplinary open access archive for the deposit and dissemination of scientific research documents, whether they are published or not. The documents may come from teaching and research institutions in France or abroad, or from public or private research centers.
L'archive ouverte pluridisciplinaire HAL, est destinée au dépôt et à la diffusion de documents scientifiques de niveau recherche, publiés ou non, émanant des établissements d'enseignement et de recherche français ou étrangers, des laboratoires publics ou privés. 


\title{
Transition to chaotic vibrations for harmonically forced perfect and imperfect circular plates
}

\author{
C. Touzéa,*, O. Thomas ${ }^{\mathrm{b}}$, M. Amabilic \\ a ENSTA-UME, Unité de Mécanique, Chemin de la Hunière, 91761 Palaiseau Cedex, France \\ b CNAM-LMSSC, Laboratoire de Mécanique des Structures et des Systèmes Couplés, 2 rue Conté, Paris, France \\ ${ }^{\mathrm{c}}$ Department of Mechanical Engineering, McGill University, 817 Sherbrooke Street West, Montreal, Canada
}

\begin{abstract}
A B S T R A C T
The transition from periodic to chaotic vibrations in free-edge, perfect and imperfect circular plates, is numerically studied. A pointwise harmonic forcing with constant frequency and increasing amplitude is applied to observe the bifurcation scenario. The von Kármán equations for thin plates, including geometric non-linearity, are used to model the large-amplitude vibrations. A Galerkin approach based on the eigenmodes of the perfect plate allows discretizing the model. The resulting ordinary-differential equations are numerically integrated. Bifurcation diagrams of Poincaré maps, Lyapunov exponents and Fourier spectra analysis reveal the transitions and the energy exchange between modes. The transition to chaotic vibration is studied in the frequency range of the first eigenfrequencies. The complete bifurcation diagram and the critical forces needed to attain the chaotic regime are especially addressed. For perfect plates, it is found that a direct transition from periodic to chaotic vibrations is at hand. For imperfect plates displaying specific internal resonance relationships, the energy is first exchanged between resonant modes before the chaotic regime. Finally, the nature of the chaotic regime, where a high-dimensional chaos is numerically found, is questioned within the framework of wave turbulence. These numerical findings confirm a number of experimental observations made on shells, where the generic route to chaos displays a quasiperiodic regime before the chaotic state, where the modes, sharing internal resonance relationship with the excitation frequency, appear in the response.
\end{abstract}

\section{Introduction}

When subjected to intensive and large-amplitude external forcing, thin plates and shells can easily experience chaotic vibrations. The study of the transition from periodic to chaotic vibrations is of primary interest in numerous applied fields such as aeronautic and aerospace or civil engineering, where shell-like structural components are often used [1,2]. Another field where chaotic vibration of shells is searched for, is that of musical acoustics and more precisely the sound of gongs and cymbals where the chaotic nature of the vibration ensures for the peculiar bright and shimmering sound of these instruments [3-8]. A convenient and reproducible way to experimentally study the chaotic vibrations of gongs and cymbals consists in harmonically forcing the structure with an increasing amplitude. Numerous experiments on different cymbals and gongs have been performed and are reported in $[9,7,10,8,11,12]$. The generic observation, that is also valid for plates and shells, reveals a scenario for the transition to chaotic vibrations including two bifurcations, separating three distinctive regime. Fig. 1 shows two typical spectrograms of recorded pointwise velocity (measured with a laser vibrometer) for an experiment with increasing amplitude of forcing. Fig. 1(a) shows the case of a cymbal of diameter $55.6 \mathrm{~cm}$ excited at $\Omega=467 \mathrm{~Hz}$, while in Fig. 1(b) a smaller cymbal of diameter $30.4 \mathrm{~cm}$ is excited at $1151 \mathrm{~Hz}$. For small amplitude values of the forcing, a periodic regime is at hand. When the excitation frequency is close to an eigenfrequency, a linear resonance occurs, large-amplitude motions are more easily observable, resulting in the appearance of harmonics of the forcing frequency. For moderate amplitudes, a bifurcation occurs and a quasiperiodic regime is observed. This quasiperiodic state is characterized by the appearance of a number of distinct frequency peaks in the spectrum, each of them being an eigenmode of the structure. Moreover, these new frequency peaks can be grouped by two or three, and they present simple non-linear resonance relationships with the excitation frequency $[9,7,10,8]$. Hence, this transition is interpreted as a loss of stability of the first excited mode, in favour of a coupled regime, involving all the modes that share an internal resonance relationship with the excitation frequency, the injected energy being spread all over this specific set of modes. Finally, for high values of the forcing amplitude, a 

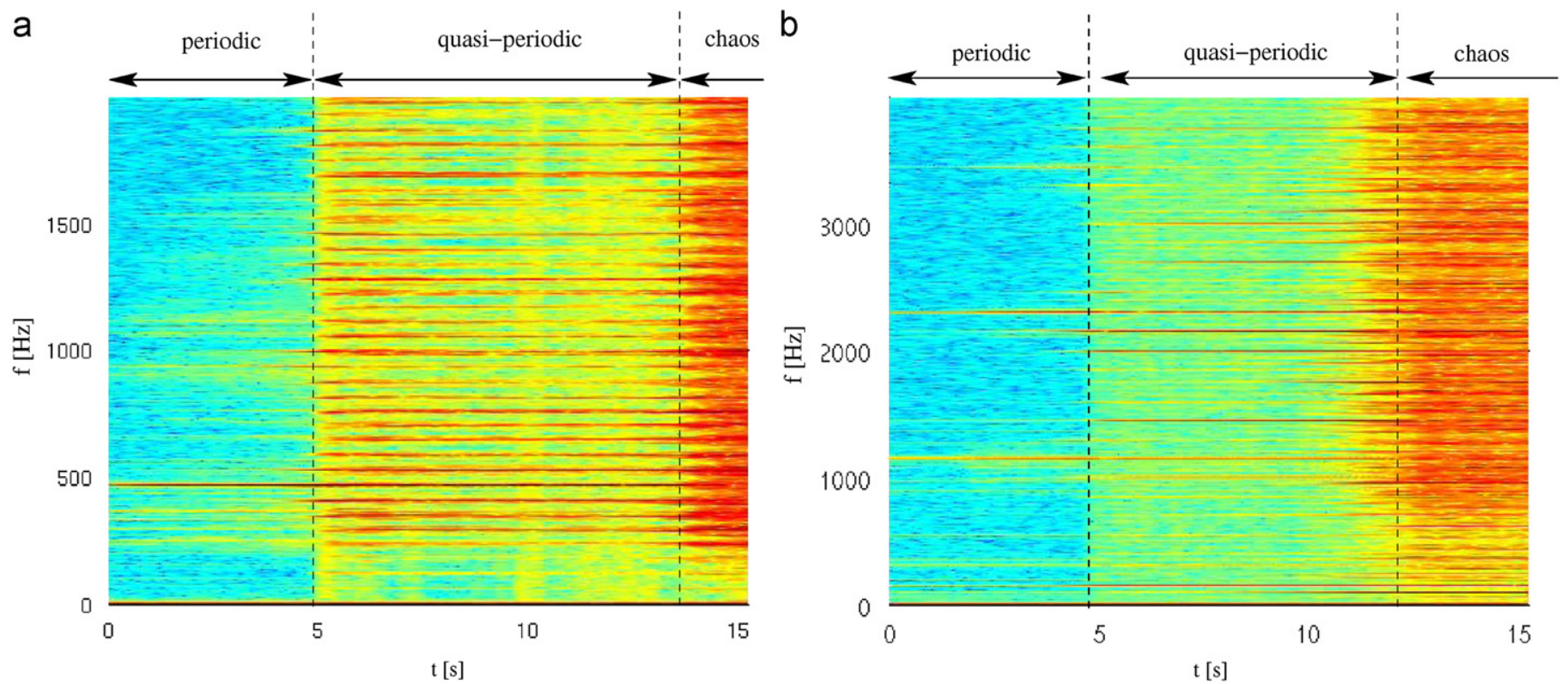

Fig. 1. (color online) Spectrograms of the vibration of two different cymbals excited harmonically with a linearly increasing amplitude. (a) cymbal of diameter $55.6 \mathrm{~cm}$, thickness $1.3 \mathrm{~mm}$, excited at $467 \mathrm{~Hz}$. (b) cymbal of diameter $30.4 \mathrm{~cm}$, thickness $0.7 \mathrm{~mm}$, excited at $1151 \mathrm{~Hz}$. In each case the three different vibration regimes are clearly identified.

second bifurcation occurs and a chaotic vibration, characterized by a broadband Fourier spectrum and a positive Lyapunov exponent, sets in $[7,8]$.

From the numerical viewpoint, the transition from periodic to chaotic vibrations in continuous systems has been considered by Awrejcewicz et al. in a series of papers [13-16], with various numerical methods (finite-difference and Bubnov-Galerkin method) and for plates, cylindrical shells, panels and sector-type spherical shells. No confrontations to experiments were reported, but a modified Ruelle-Takens-Newhouse scenario is found in [16] which closely resembles our experimental results. Amabili studied the transition to chaotic vibrations for circular cylindrical shells and doubly curved panels $[17,18,2]$, but only in the vicinity of the fundamental frequency. The dynamic instability and chaotic vibration occurring near the buckling critical load are also investigated in [19]. Comparisons of experimental and theoretical results are provided by Murphy et al. for a plate subjected to large-amplitude acoustical excitation [20]. Amongst other things, they underline the difficulty of finding out the convergence in the dimension calculation for the underlying attractor. Nagai et al. provided very detailed analytical and experimental results for a shallow cylindrical panel, with or without a concentrated mass [21-23]. In this case, the dimension of the shell is smaller, so that a limited number of modes can be excited, and the amplitude of the forcing is kept constant while varying the frequency. Convincing experimental proofs for the presence of a low-dimensional attractor are given.

From the theoretical viewpoint, the long-time behaviour and the existence of global attractors and inertial manifolds in von Kármán dynamical equations have been studied by Chueshov and Lasiecka for various type of damping laws (viscous, structural and thermoelastic damping on the structure, or linear and non-linear boundary dissipation) [24-28]. Existence of finite-dimensional attractors with upper bounds on their Hausdorff dimension is provided [25], inertial manifolds are also shown to exist when a spectral gap condition is fulfilled, which leads to require a large amount of damping in the case of viscous dissipation [26]. On the other hand, for very lightly damped plates without strong finitesize effects, the framework of wave turbulence can be applied to study the energy repartition through lengthscales [29,30].
The case of plates have been treated recently in [31], and experimental investigations were reported in [32-34].

In this paper, the transition scenario is specifically addressed. A Galerkin method with the eigenmodes as expansion functions, is used to discretize the von Kármán plate equations. As a consequence of the number of expansion functions retained in the truncations, the scenario can be numerically tested in the vicinity of the seven first eigenfrequencies. A particular attention is paid on the existence of energy transfer between modes, that can give birth to the quasiperiodic regime occurring before the chaotic state. For perfect plates with cubic non-linearity and only order three internal resonance relationships, it is shown that no resonance relations exist for free-edge circular plates between the seven first eigenfrequencies. Consequently, as shown in Section 3, a direct transition to chaos is generically observed. Then an imperfect plate is considered, and the imperfection is selected so as to tune the first eigenfrequencies and exhibit particular order-two internal resonance. In this case, as shown in Section 4, energy exchanges are possible and the coupled regime is observed. The critical values of the force amplitude, which are needed to attain the chaotic state, are also carefully studied. Numerical results show that the imperfection significantly lowers the critical value needed to observe chaotic vibration, which is fully consistent with the experimental observations. Finally, the convergence of the Galerkin truncation is systematically addressed, with respect to the critical force, the Lyapunov exponents and the Fourier spectra of vibration, leading to a discussion of the results obtained in the chaotic state, with regard to the occurrence of wave turbulence or low-dimensional chaos.

\section{Model equations}

\section{1. von Kármán equations for perfect and imperfect plates}

The dynamic analog of the von Kármán equations for thin plates is used to model the large-amplitude vibrations. The main assumptions of the model rely in the strain/displacement relationship, where only the leading term depending on the transverse displacement $\bar{w}$, is retained $[2,35,36]$. In the remainder 
of the paper, bar-variables (such as $\bar{w}$ ) refer to dimensional quantities. The bars will be omitted when non-dimensionalization will be realized. In-plane and rotary inertia are neglected, so that an Airy stress function $\bar{F}$ can be used. An imperfect circular plate of diameter $2 a$, thickness $h$, made of an homogeneous isotropic material of density $\rho$, Poisson's ratio $v$ and Young's modulus $E$ is considered. A static imperfection associated with zero initial stress is denoted as $\bar{w}_{0}$. The local equations for the imperfect plate reads [37-39]:

$D \Delta \Delta \bar{w}+\rho h \ddot{\bar{w}}=L(\bar{w}, \bar{F})+L\left(\bar{w}_{0}, \bar{F}\right)-\bar{c} \dot{\bar{w}}+\bar{p}$,

$\Delta \Delta \bar{F}=-\frac{E h}{2}\left[L(\bar{w}, \bar{w})+2 L\left(\bar{w}, \bar{w}_{0}\right)\right]$,

where $\bar{w}$ represents the transverse displacement from the deformed static configuration, $\bar{c}$ is a damping coefficient, $\bar{p}$ represents the external distributed forcing, and $D$ is the flexural rigidity. The bilinear operator $L$ writes, in polar coordinates:

$$
\begin{aligned}
& L(\bar{W}, \bar{F})=w_{, \overline{r r}}\left(\frac{\bar{F}_{, \bar{r}}}{\bar{r}}+\frac{\bar{F}_{, \theta \theta}}{\bar{r}^{2}}\right)+\bar{F}_{, \overline{r r}}\left(\frac{\bar{w}_{, \bar{r}}}{\bar{r}}+\frac{\bar{w}_{, \theta \theta}}{\bar{r}^{2}}\right) \\
& -2\left(\frac{\bar{w}_{, \bar{r} \theta}}{\bar{r}}-\frac{\bar{w}_{, \theta}}{\bar{r}^{2}}\right)\left(\frac{\bar{F}_{, \bar{r} \theta}}{\bar{r}}-\frac{\bar{F}_{, \theta}}{\bar{r}^{2}}\right) \text {. }
\end{aligned}
$$

When $\bar{w}_{0}=0$ (no imperfection), one recovers the usual von Kármán equations for perfect plates [40]. A free-edge boundary condition is considered, which enforces the following relationships to be fulfilled, for all $\bar{t}$ and $\theta$ :

$\bar{F}$ and $\bar{w}$ are bounded at $\bar{r}=0$,

$\bar{F}_{, \bar{r}}+\frac{1}{a} \bar{F}_{, \theta \theta}=0, \quad \bar{F}_{, \bar{r} \theta}+\frac{1}{a} \bar{F}_{, \theta}=0 \quad$ at $\bar{r}=a$,

$\bar{w}_{, \overline{r r}}+\frac{v}{a} \bar{w}_{, \bar{r}}+\frac{v}{a^{2}} \bar{w}_{, \theta \theta}=0 \quad$ at $\bar{r}=a$,

$\bar{w}_{, r r r}+\frac{1}{a} \bar{w}_{, \overline{r r}}-\frac{1}{a^{2}} \bar{w}_{, \bar{r}}+\frac{2-v}{a^{2}} \bar{w}_{, \bar{r} \theta \theta}-\frac{3-v}{a^{3}} \bar{w}_{, \theta \theta}=0 \quad$ at $\bar{r}=a$.

Eqs. (1)-(3) are made non-dimensional by introducing the following quantities:

$\bar{w}=h w, \quad \bar{w}_{0}=h w_{0}, \quad \bar{r}=a r$,

$\bar{F}=E h^{3} F, \quad \bar{t}=\sqrt{\frac{\rho h a^{4}}{D}} t$,

$\bar{p}=\frac{E h^{4}}{\varepsilon a^{4}} p, \quad \bar{c}=\frac{E h^{3}}{2 \varepsilon a^{2}} \sqrt{\frac{\rho h}{D}} 4 \mu$,

with $\varepsilon=12\left(1-v^{2}\right)$.

Substituting the above definitions in Eqs. (1), non-dimensional equations of motion of imperfect plates with free-edge and subjected to large deflection finally writes, in terms of the non-dimensional variables:

$\Delta \Delta w+\ddot{w}=\varepsilon\left[L(w, F)+L\left(w_{0}, F\right)\right]-2 \mu \dot{w}+p$,

$\Delta \Delta F=-\frac{1}{2}\left[L(w, w)+2 L\left(w, w_{0}\right)\right]$.

All the numerical analysis of the next sections will be made on the non-dimensional equations, without referring to a particular plate, so that the study focuses now on the variables without a bar.

\subsection{Modal projection}

The equations of motion (5) are discretized by expanding $w, w_{0}$ and $F$ onto the eigenmodes of the perfect plate, i.e. the modes of the linear system associated to Eqs. $(5 \mathrm{a}, \mathrm{b})$ with $w_{0}=0$, via:

$w_{0}(r, \theta)=\sum_{p=1}^{N_{0}} a_{p} \Phi_{p}(r, \theta)+z_{g}$,

$w(t, r, \theta)=\sum_{p=1}^{N} q_{p}(t) \Phi_{p}(r, \theta)$,

$F(t, r, \theta)=\sum_{p=1}^{N_{F}} \eta_{p}(t) \Psi_{p}(r, \theta)$

where $z_{g}$ is the center of mass' offset, $\left\{a_{p}\right\}_{p=1 \ldots N_{0}}$ represent the projection of the imperfection onto the eigenmodes of the perfect plate, and $\left\{q_{p}\right\}_{p=1 \ldots N}$, the modal amplitudes for the vibratory part, which will be our main unknown in the remainder of the study. In the above equations, the $\left\{\Phi_{i}\right\}_{i \in \mathbb{N}}$ are the transverse vibration mode shapes of the perfect plate and the $\left\{\Psi_{i}\right\}_{i \in \mathbb{N}}$ are membrane modes, defined by, for all $i \in \mathbb{N}^{*}$ :

$\Delta \Delta \Phi_{i}-\omega_{i}^{2} \Phi_{i}=0$,

$\Delta \Delta \Psi_{i}-\zeta_{i}^{4} \Psi_{i}=0$

together with boundary conditions (3a,c,d) for $\Phi_{i}$ and (3a,b) for $\Psi_{i}$. In the above equations, $\omega_{i}$ is the $i$-th non-dimensional natural flexural frequency of the perfect plate. The analytical expressions for $\Phi_{i}$ and $\Psi_{i}$ as well as numerical values for $\omega_{i}$ and $\zeta_{i}$ can be found in [40]. The critical parameters in such Galerkin expansions are the number of retained mode in the numerical analysis. In this study, the number of in-plane modes has been fixed to 12 , a sufficient value to ensure a five-digits accuracy for the cubic non-linear coefficients up to the 15th modes, and four-digits accuracy up to the 26th modes [40,41]. This accuracy is sufficient for the truncations we will study in the remainder of the paper. The imperfections that will be considered in Section 4 have the shape of the first axisymmetric mode, so that we will have $N_{0}=1$. Finally, the number of transverse modes $N$ is kept variable so as to carefully study the convergence of the results. Assuming normalization of the modes and applying a usual projection technique, one finally obtains the following dynamical equations governing the evolution of the modal amplitudes, for all $u \in[1, N]$ :

$$
\begin{aligned}
\ddot{q}_{u}+\omega_{u}^{2} q_{u}= & -\varepsilon\left[\sum_{p=1}^{N} \alpha_{p}^{u} q_{p}+\sum_{p=1}^{N} \sum_{r=1}^{N} \beta_{p r}^{u} q_{p} q_{r}\right. \\
& \left.+\sum_{p=1}^{N} \sum_{r=1}^{N} \sum_{s=1}^{N} \Gamma_{r s p}^{u} q_{p} q_{r} q_{s}\right]-2 \mu \dot{q}_{u}+p_{u} .
\end{aligned}
$$

Expressions for $p_{u}$ and $\Gamma_{r s p}^{u}$ are

$p_{u}=\iint_{S} \Phi_{u}(r, \theta) p(r, \theta, t) \mathrm{d} S$

$\Gamma_{r s p}^{u}=-\frac{1}{2} \sum_{q=1}^{N_{F}} \frac{1}{\zeta_{q}^{4}} \iint_{S} \Phi_{u} L\left(\Phi_{p}, \Psi_{q}\right) \iint_{S} \Psi_{q} L\left(\Phi_{r}, \Phi_{s}\right) \mathrm{d} S$.

The cubic coefficients $\Gamma_{r s p}^{u}$ are those of the perfect case, presented in [40]. The linear and quadratic coupling coefficients $\alpha_{p}^{u}$ and $\beta_{p r}^{u}$ appearing in Eq. (8) stem from the geometric imperfection $w_{0}$, and are thus expressed as functions of the amplitudes $a_{p}$ of the 
expansion of $w_{0}$ introduced in Eq. (6a). They write [37]

$\alpha_{p}^{u}=-\sum_{r=1}^{N_{0}} \sum_{s=1}^{N_{0}} 2 \Gamma_{r p s}^{u} a_{r} a_{s}$

$\beta_{p r}^{u}=-\sum_{s=1}^{N_{0}}\left(\Gamma_{r p s}^{u}+2 \Gamma_{s r p}^{u}\right) a_{s}$

\subsection{Numerical details}

In the remainder of the paper, a dimensional pointwise harmonic forcing of magnitude $\bar{F}$ (in N) and frequency $\bar{\Omega}$ (in rad/s), located at $\left(r_{0}, \theta_{0}\right)$, is considered, thus $\bar{p}(\bar{r}, \theta, \bar{t})=\delta\left(\bar{r}-\bar{r}_{0}\right) \delta\left(\theta-\theta_{0}\right) \bar{F} \cos \bar{\Omega} \bar{t}$. Following Eqs. (4)-(9a), the non-dimensional forcing on Eq. (8) reads

$p_{u}=\Phi_{u}\left(r_{0}, \theta_{0}\right) F \cos \Omega t \quad$ with $F=\frac{\varepsilon a^{4}}{E h^{4}} \bar{F} \quad$ and $\quad \Omega=\frac{a^{2}}{h} \sqrt{\frac{12 \rho\left(1-v^{2}\right)}{E}} \bar{\Omega}$.

In order to simulate the response of lightly damped plates, which is generally the case for a metallic material such as brass or steel, the damping coefficient has been set to $\mu=0.002$. The non-linear cubic coefficients are computed once and for all from the analytical expressions, and are stored in a subsequent four-dimensional array. The non-dimensional eigenfrequencies $\omega_{k}$ for the perfect plate are recalled in Table 1 . The modes are classified, as it is usual for circular plates, with two indexes $(k, n), k$ being the number of nodal diameters and $n$ the number of nodal circles. Modes $(0, n)$ are called axisymmetric, while $k \neq 0$ implies an asymmetric mode, which have two companion (or preferential) configurations for the same eigenfrequency [40,37]. For asymmetric modes, a binary index is often added in order to distinguish the two configurations, say $(2,0, C)$ for the cosine mode and $(2,0, S)$ for the sine configuration. Hence each column corresponding to an asymmetric mode in Table 1 has dimension two, for the two preferential configurations.

Four truncations in the Galerkin expansion (6b) are retained in the numerical simulations. The first one contains $N=10$ modes: $(2,0),(0,1),(3,0),(1,1),(4,0)$ and $(0,2)$. In each truncation, the eigenmodes are retained by increasing values of their eigenfrequencies. When the last asymmetric mode is selected, one (or two) axisymmetric modes are added in order to ensure a good stability of the model, due to the well-known coupling between asymmetric and axisymmetric motions [42-44]. This is also why in Table 1, eigenmodes are presented by increasing order of the frequencies, except the last two (axisymmetric), which have significantly larger eigenfrequencies: asymmetric modes in between have not been reported in the table. The second truncation contains $N_{w}=19$ modes. Modes $(5,0),(2,1),(6,0)$, $(0,3),(0,4)$ and $(0,5)$ are added to the previous 10 modes truncation. A 27 modes model is considered by adding modes $(3,1),(1,2),(7,0)$ and $(4,1)$, and a 35 modes truncation will also be selected by adding $(8,0),(2,2),(5,0)$ and $(9,0)$.

The resulting ordinary-differential equations (ODEs) governing the dynamics, Eq. (8), are numerically integrated by using the DIVPAG routine of the Fortran library IMSL, where a variable-order method based on backward differentiation formulas (BDFs), also known as Gear's BDFs, is implemented. The Gear's BDFs method is a multistep, implicit method especially designed for stiff problems $[45,46]$. In our case, the dynamical solutions are particularly difficult to integrate, because of the large dimension of the phase space, the stiff character of the simulated dynamics, the very low value of the damping, and the bifurcations, implying finally a chaotic state with an attractor of large dimension. In particular, explicit Runge-Kutta methods, leap-frog scheme (also known as Störmer-Verlet [47]), as well as all the algorithm of the Matlab ODE suite [48], have been tested, all of them giving divergence due to numerical instability.

For analyzing the results when varying the amplitude of the external forcing, three usual techniques are used. First, stroboscopic Poincaré maps at the excitation frequency (with a zero phase) will permit detection of the different regimes (periodic, quasiperiodic and chaotic). Power spectra of the transverse velocity will be used to analyze the chaotic motions in Section 3.5. Finally Lyapunov exponents will be computed for reviewing the presence of chaos, using the numerical procedures described in $[2,18]$.

\section{Simulation results for the perfect plate}

In this section the numerical results on the transition from periodic to chaotic vibration for a perfect plate, are considered. The plate is harmonically forced with fixed $\Omega$ and increasing $F$. Section 3.1 shows the generic result obtained, for excitation frequencies $\Omega$ between 1.5 and 25. The convergence vs the critical value $F_{c r}$ needed to attain the chaotic regime is shown in Section 3.2 , and the complete bifurcation diagram $F$ vs $\Omega$ is given in Section 3.3. Some particular cases are shown in Section 3.4. Finally, Lyapunov exponents and power spectra are shown in Section 3.5, which leads to discuss the nature of the chaotic regime and to reconsider the convergence for the chaotic solution.

\subsection{Generic result}

The simulations conducted aims at finding the stability limits of forced periodic orbits in the vicinity of the first eigenfrequencies. Fig. 2 shows the response of four modal coordinates, $q_{(2,0, C)}$, $q_{(2,0, S)}, q_{(0,1)}$ and $q_{(3,0, C)}$, for an excitation frequency of $\Omega=5.3$, and an increasing value of the non-dimensional forcing from 0 to 20 . The excitation frequency has been chosen close to $\omega_{(2,0)}$, and the point of excitation is located at $r=1, \theta=0$, so that only the cosine configuration is excited. The model with 19 modes is selected for this simulation. Each time the value of the forcing is increased (with a step of 0.03 ), a long transient of 5000000 of periods is waited, then 1000000 of periods are recorded for the Poincaré section. As the upper stability limit is sought, particular attention must be paid in the numerical simulations: the force amplitude must be very slowly increased step by step, so as not to jump in the chaotic regime because of a large perturbation given to the system. Long transients are needed because of the low damping value, so that each run is particularly time-consuming. A typical

Table 1

Non-dimensional frequencies of a perfect circular plate with a free-edge, by increasing order.

\begin{tabular}{|c|c|c|c|c|c|c|c|c|c|c|}
\hline Mode & $(2,0)$ & $(0,1)$ & $(3,0)$ & $(1,1)$ & $(4,0)$ & $(5,0)$ & $(2,1)$ & $(0,2)$ & $(6,0)$ & $(3,1)$ \\
\hline$\omega_{k}$ & 5.26 & 9.06 & 12.24 & 20.51 & 21.52 & 33.06 & 35.24 & 38.51 & 46.81 & 52.92 \\
\hline Mode & $(1,2)$ & $(7,0)$ & $(4,1)$ & $(8,0)$ & $(2,2)$ & $(0,3)$ & $(5,1)$ & $(9,0)$ & $(0,4)$ & $(0,5)$ \\
\hline$\omega_{k}$ & 59.86 & 62.73 & 73.37 & 80.83 & 84.37 & 87.81 & 96.50 & 101.07 & 156.88 & 245.69 \\
\hline
\end{tabular}

The first 18th frequencies are given, then the two next axisymmetric modes are included in the table, as being present in the retained truncations for stability. 

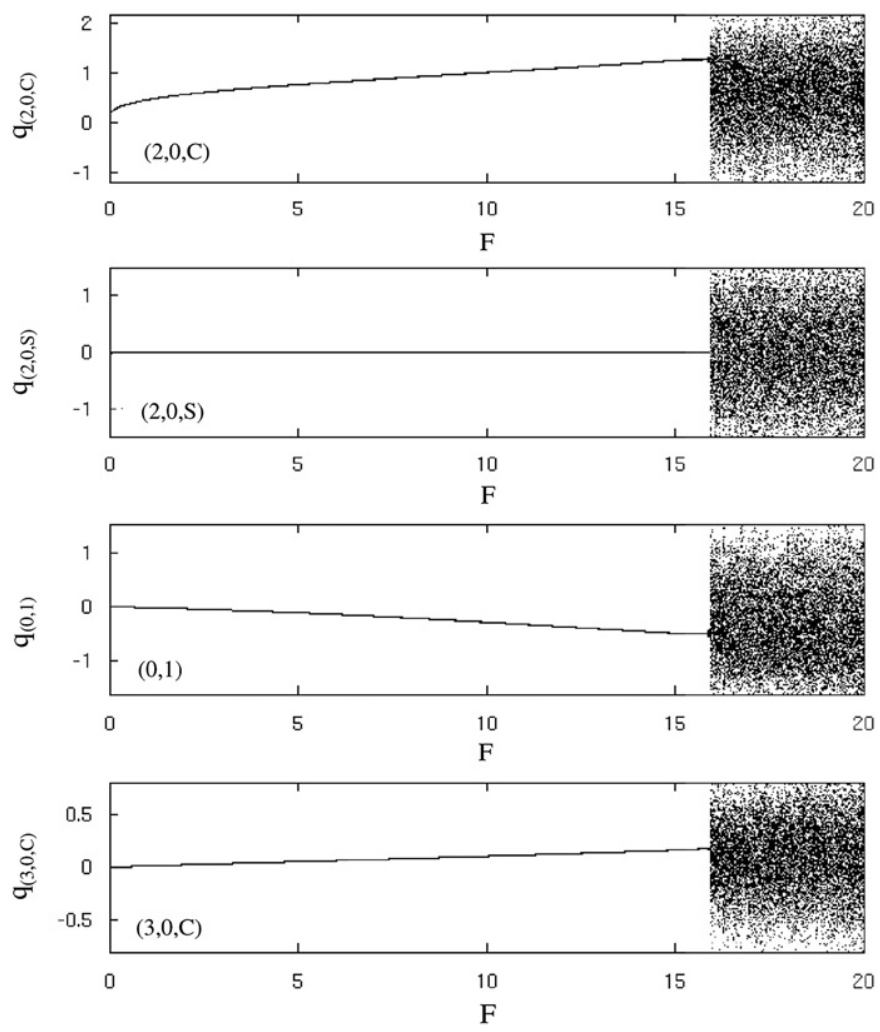

Fig. 2. Bifurcation diagram of Poincare section for the first four modes of a perfect plate, excited at $\Omega=5.3$, with an increasing $F$ from 0 to 20. From top to bottom: modes $(2,0, \mathrm{C}),(2,0, \mathrm{~S}),(0,1)$ and $(3,0, \mathrm{C})$. A periodic regime is observed until $F=F_{c r}=15.92$, where a chaotic vibration sets in.

run shown in Fig. 2 took around $20 \mathrm{~h}$ of computation on a standard PC with a CPU-clock at $2 \mathrm{GHz}$.

In Fig. 2, which is typical of the results, a periodic response is obtained until $F=F_{c r}=15.92$, where the chaotic regime suddenly sets in with energy on all the modal amplitudes $\left\{q_{i}\right\}_{i=1 \ldots 1}$. Before the chaotic regime, non-zero (though small) values for modes $(0,1)$ and $(3,0, C)$ are observed. These are due to non-resonant couplings between the linear modes, that bends the non-linear normal mode (NNM) corresponding to the mode $(2,0, \mathrm{C})$ that is directly excited [49]. As the invariant manifold is curved and the periodic orbits computed are contained within it, small non-zero values are obtained for some modal amplitudes. However, a strong coupling is not at hand, and the regime is periodic along the NNM corresponding to $(2,0, C)$. In the chaotic regime, the amplitudes of low-frequency modes are larger than the others, more precisely the amplitudes of the modal coordinates decrease continuously with frequency.

From Eq. (11), the relationship between the non-dimensional force $F$ reported in Fig. 2 and its dimensional counterpart $\bar{F}$ reads $\bar{F}=\left(E h^{4} / \varepsilon a^{4}\right) F$. For a typical circular plate having the dimension of a cymbal, i.e. with $E=110 \mathrm{GPa}, \quad h=1 \mathrm{~mm}, \quad a=0.2 \mathrm{~m}$ and $\varepsilon=12\left(1-v^{2}\right)$ with $v=0.33$, the critical non-dimensional force $F_{c r}=15.92$ leads to a dimensional forcing $\bar{F}=102.35 \mathrm{~N}$, which is significantly larger than the experimental values needed to obtain chaotic behaviour, which are of the order of $2-10 \mathrm{~N}$ [8,50]. In Section 4, it will be shown that considering an imperfection (unavoidable in real plates) significantly reduces this critical value to those observed experimentally.

The result shown in Fig. 2 is generic in the sense that numerous simulations have been performed for $\Omega$ varying between 1.5 and 25 , and in quite all cases a direct transition
Table 2

Value of the critical force $F_{c r}$ needed to observe the chaotic regime, for three different excitation frequencies $\Omega=5.3,9.4$ and 21.2, and for eight different truncations with an increasing number of modes $N$.

\begin{tabular}{lllllllll}
\hline$N$ & 2 & 3 & 5 & 7 & 10 & 19 & 27 & 35 \\
\hline$\Omega=5.3$ & 75.9 & 15.80 & 16.03 & $\times$ & 15.88 & 15.95 & 15.95 & $\times$ \\
$\Omega=9.4$ & $/$ & $/$ & 14.54 & 12.49 & 12.29 & 12.19 & 12.24 & $\times$ \\
$\Omega=21.2$ & $/$ & $/$ & $/$ & $/$ & 30.24 & 9.17 & 10.29 & 10.1 \\
\hline
\end{tabular}

The symbol / means that chaos was not observed until very large values of $F$, and $\times$ means that the simulation has not been realized.

from periodic to chaotic motion has been observed. This will be reconsidered in Section 3.3.

\subsection{Convergence study}

Before entering more deeply in the analysis of the numerical results, a convergence study is in order. Here, the convergence of the Galerkin expansions is checked with respect to the critical force $F_{c r}$ needed to attain the chaotic regime. Table 2 sums up the convergence study, showing $F_{c r}$ obtained for three different frequencies $\Omega$ tested, and for an increasing number of modes $N$ retained in the truncation for the transverse displacement, see Eq. (6b). The results show that the model with 19 modes captures the good value of $F_{c r}$ in the three cases. More surprisingly, the convergence study highlights the fact that a very limited number of modes are needed for obtaining a converged value for $F_{c r}$. Only three modes are necessary for getting the critical force when exciting around the first eigenfrequency, namely $(2,0, \mathrm{C}),(2,0, \mathrm{~S})$ and $(0,1)$. This result underlines once again the importance of axisymmetric modes. With only the two companion configuration $(2,0, \mathrm{C})$ and $(2,0, \mathrm{~S})$, in $1: 1$ internal resonance, another bifurcation is found with a much larger value of $F=75.9$. Hence the dynamics for the transition to chaos in the vicinity of the first mode is completely determined by the first three modes. When $\Omega=9.4$, i.e. in the vicinity of mode $(0,1)$, seven modes are necessary to get the correct result. The number of involved modes increases logically as we are obliged to include all the modes that have an eigenfrequency smaller than the excitation. However, only a very few modes over the excitation frequency are needed to get convergence.

These results indicates that the transition to the chaotic vibration is completely governed by the slow-flow equations, i.e. the low-frequency part of the dynamics. The fast-flow dynamics has no influence on the determination of $F_{c r}$ and can be discarded. From a phase-space point of view, the numerical experiment studied here can be seen as searching the stability limit of the NNM in the vicinity of the eigenfrequencies of the structure. A NNM being composed of periodic orbits, by increasing the force we move away from the stable origin until the limit of the stable manifold is attained. Then the chaotic motion, also referred to as global stochasticity for Hamiltonian systems [51], is observed. Our numerical results show that the geometry and the limiting stable periodic orbits are completely determined by the slow-flow modes.

However, once the chaotic state established, all the modal coordinates have a significant amplitude, as already noted in Fig. 2, which means that the energy is spread all over the modes. Hence a major effect of increasing the number of modes is to lower the response amplitude of the slow-flow modes. Fig. 3 shows the amplitude of mode $(0,1)$ for the same experiment with $\Omega=5.3$, and conducted with $N=3$ and 27 . For $N=3$, all the energy is spread between three modes and $(0,1)$ has a very large amplitude. This amplitude is continuously decreased when modes 
are added in the truncation, showing that there is a flux of energy from low to high-frequency modes. This cascade of energy in the spectral domain leads to a decrease of the amplitudes of the slowflow modes, as shown in Fig. 3. This result indicates that the numerical solution is probably not converged in the chaotic regime. This will be more deeply investigated in Section 3.5, where the wave turbulence framework will allow for a better understanding of the chaotic regime.

\subsection{Bifurcation diagram}

The complete bifurcation diagram $F_{c r}$ vs $\Omega$ is given in Fig. 4, for $\Omega \in[1.5,25]$. Each point represents a numerical experiments
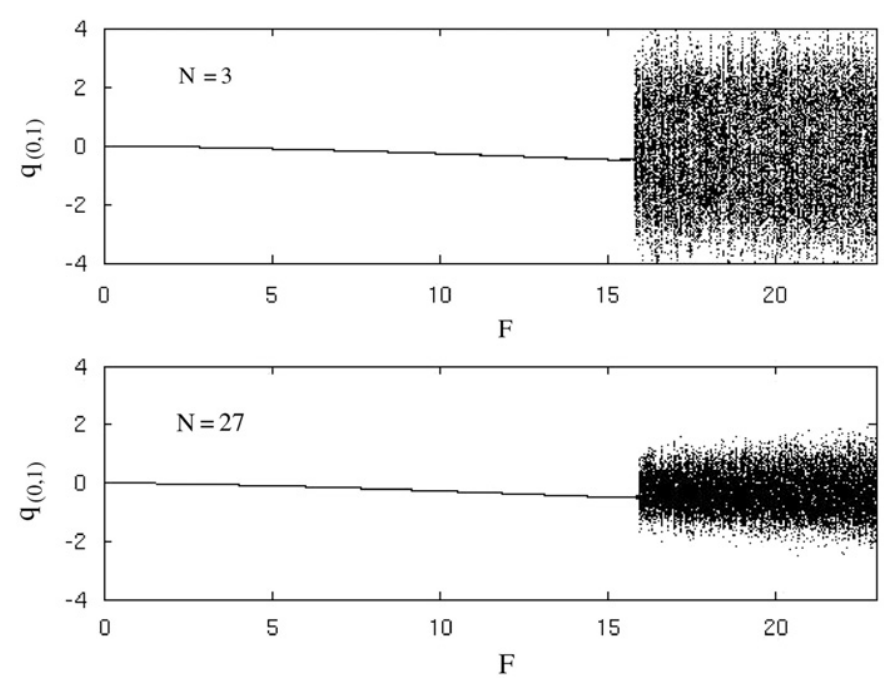

Fig. 3. Bifurcation diagram of Poincaré maps for mode $(0,1)$, excited at $\Omega=5.3$ with $F \in[0,20]$. Top: with a three modes model. Bottom: with a 27 modes model. equivalent to the one shown in Fig. 2, where the value $F_{c r}$ has been reported. On the diagram are represented the eigenfrequencies of $(2,0),(0,1),(3,0),(1,1)$ and $(4,0)$. As the perfect plate displays only cubic non-linearity, only order-three resonances are supposed to come into play. Hence the $1 / 3$ subharmonics have also been represented in thin dashed lines, as well as the only superharmonic of order three: $3 \omega_{(2,0)}=15.78$. The diagram has been obtained with the model composed of 19 modes, except from the frequency $\Omega=16$ where the 27 modes truncation has been used. Effectively, from $\Omega=16$ appears the $1 / 3$ subharmonics of modes $(3,1)\left(\omega_{(3,1)} / 3=17.64\right),(1,2)\left(\omega_{(1,2)} / 3=19.95\right)$ and $(7,0)$ $\left(\omega_{(7,0)} / 3=20.91\right)$ that have to be included in the truncation.

Fig. 4 shows that the critical values are generally large, except around 6.6 and in the interval $[20.5,21.5]$ where two minimums are observed with $F_{c r}$ around 5 and 3, values that are close to the experimental ones. Each subharmonic creates a short tongue where the resonance give rise to a lower $F_{c r}$, which is particularly evident before the first eigenfrequency. In the linear resonance regions, i.e. in the vicinity of the eigenfrequencies, the minimum value of $F_{c r}$ is observed at a higher value of excitation frequency than the eigenfrequency. This is a consequence of the hardening character of the plate: frequency-response curves in forced regime are bended to the higher frequencies. For example the region where mode $(2,0)$ is directly excited corresponds to the tongue that extends up to $\Omega=6.6$. The value of $F_{c r}$ decreases when $\Omega$ is increased from 5.26 to 6.6: as long as the system is able to catch the upper branch of the frequencyresponse curve by jump phenomenon, large-amplitude motions are more easily attained and chaos is observed for a smaller value of $F_{c r}$. The same reasoning holds for the other frequencies; the tongue corresponding to mode $(0,1)$ hence extends to $\Omega=10$, and the one for mode $(3,0)$ extends to $\Omega=14.7$.

As stated in Section 3.1, all the tested frequencies $\Omega$ show a direct transition from periodic to chaotic motion, without an intermediate stage of quasiperiodic motion with a significative coupling between internally resonant modes. The most important order-three internal resonances involving the first 10 modes in

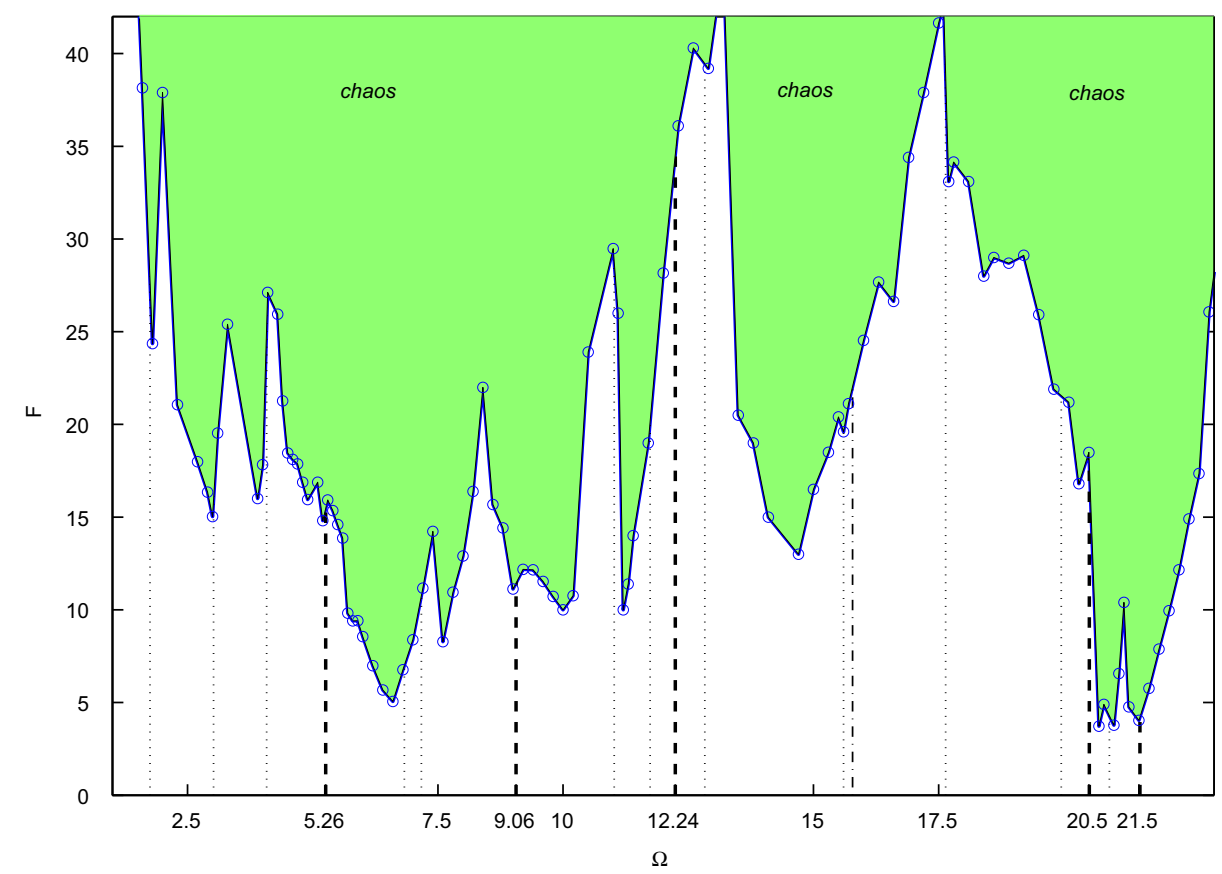

Fig. 4. Complete bifurcation diagram $F$ vs $\Omega$ for a perfect circular plate with a free-edge. The gray region (green with online colors) stands for chaotic regime, below periodic regime are found. Thick dashed lines indicate the eigenfrequencies, thin dotted lines the $1 / 3$ subharmonics and thin dashed line the third superharmonics of the eigenfrequencies. (For interpretation of the references to color in this figure legend, the reader is referred to the web version of this article.) 
the circular plate are the following:

$-\omega_{(3,0)}+\omega_{(0,2)}-\omega_{(6,0)}+\omega_{(1,1)}=-0.03$,

$-\omega_{(4,0)}+\omega_{(2,1)}-\omega_{(6,0)}+\omega_{(5,0)}=-0.03$,

$-\omega_{(0,1)}+\omega_{(4,0)}-\omega_{(5,0)}+\omega_{(1,1)}=-0.09$,

$-\omega_{(0,1)}+\omega_{(2,1)}-\omega_{(0,2)}+\omega_{(3,0)}=-0.09$.

As already noted by Nayfeh [52], when internal resonances are present, the energy transfer is from the high to the low frequencies, which means that in order to observe a modal coupling and a quasiperiodic regime, one must excite a mode involved in an internal resonance relationship with lower frequency modes. In Eqs. (12), at least two modes of higher frequencies than the tested range are present, except for (12c), where a coupling may have been observed when exciting mode $(1,1)$. However, no mode coupling have been numerically found in the tested interval. Evidently, one should excite higher frequency modes to have a chance to excite lower frequencies internal resonance relationships. Things will be different in Section 4 where the geometric imperfection will be chosen so as to tune perfectly the very first eigenfrequencies in specific internal resonance relations.

\subsection{Particular cases}

In this section, three particular cases from the complete bifurcation diagram of Fig. 4 are shown, because they exhibit particular interesting structures before the chaotic regime. These structures have been found to occur for certain frequencies, so their existence is limited in a very narrow region of the parameter space. In that sense, some couplings are here shown but they differ radically from the modal couplings observed experimentally and that will be investigated numerically in Section 4 in the sense that they do not exist for a large band of frequency excitation and are not robust to variations.
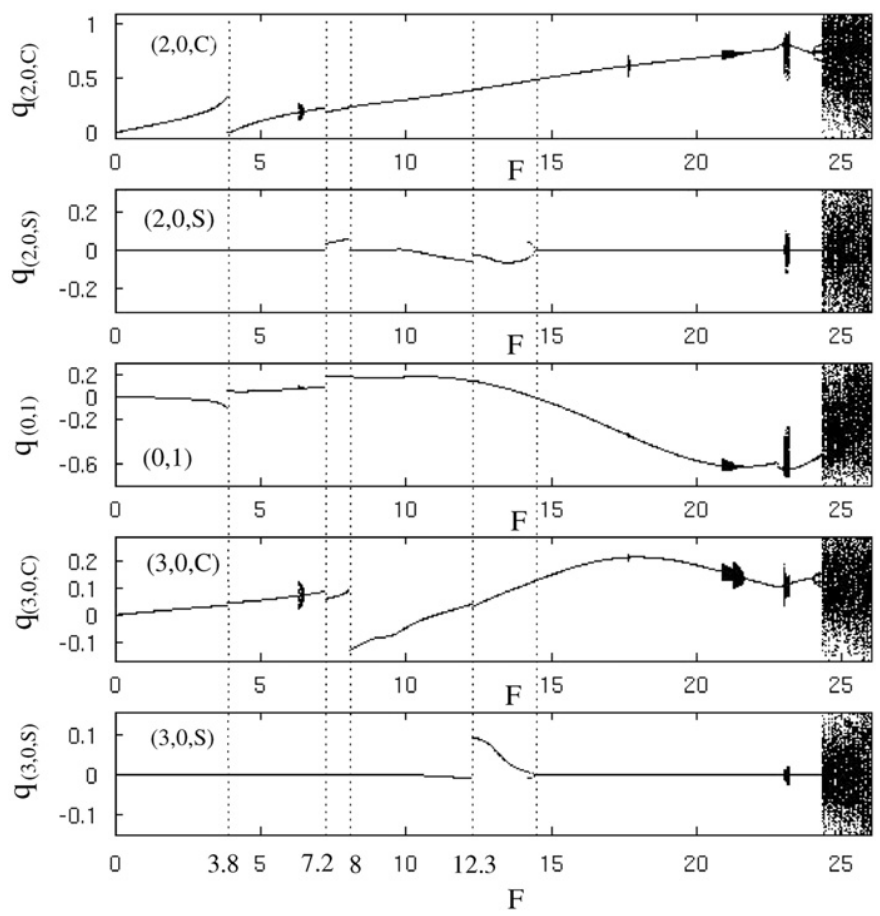

Fig. 5. Bifurcation diagram of Poincaré maps, $\Omega=1.8$ with $F \in[0,27]$.
The first case considers the $1 / 3$ subharmonic regime, obtained when forcing the perfect plate at a frequency close to one third of the first eigenfrequency $\omega_{(2,0)}$. Fig. 5 shows the bifurcation diagram of Poincaré maps obtained for $\Omega=1.8$ and $F \in[0,27]$. For $F \in[0,3.8]$, the subharmonic resonance follows the lowest branch in the Duffing-type frequency-response curve, and mode $(2,0, \mathrm{C})$ is logically the most excited one. At $F=3.9$, the jump from the low to the high-amplitude branch occurs. A coupling with $(2,0, S)$ then occurs on a very narrow region between 7.2 and 8 , then another branch is found between 12.3 and 14.4 with participation of $(2,0, S)$ and $(3,0, S)$. Note that at the end of this branch around $F=14.4$, the Poincaré section hesitates between two branches which shows that a symmetric branch exist on the whole interval, which could be chosen by the system with a slight change in the parameters. Coupling with $(0,1)$ and $(3,0, C)$ is quite strong: it is the bending of the manifold that has been already underlined in Fig. 2 for $\Omega=5$.3. Then finally chaos appears for a very high value of the forcing at $F=24.3$. This figure is quite typical of what has been observed for the first three subharmonic excitation, namely at $\Omega=1.8,3.0$ and 4.1 , with many localized events of small amplitude before the chaotic regime.

Fig. 6 shows a period-four motion that has been found for $\Omega=7.4$, for $F \in[6,10]$. This structure has not been found neither for $\Omega=7.2$, nor for 7.6, and hence exist in a very narrow region. The period-four motion exist until $F=8.7$, followed by a modulation of amplitudes. Then a periodic motion is found back. A jump phenomena occur for $F=12.6$, it is clearly visible for mode $(4,0, \mathrm{C})$ as it correspond to the jump from the low to the high-amplitude branch in the frequency response of the $1 / 3$ subharmonic motion that is here excited. Finally chaos occurs for $F=14.3$.

The last investigated particular case corresponds to $\Omega=19.8$. The bifurcation diagram of Poincaré maps has been obtained with the 27 modes truncation (the two other cases with 19 modes), and is shown in Fig. 7. This case is particularly interesting because of all the tested case, the structure that appears shares most resemblance with the sought mode coupling. The excitation frequency $\Omega=19.8$ is smaller than the eigenfrequencies of modes $(1,1)$ and $(4,0)$, which thus appears with important amplitudes in
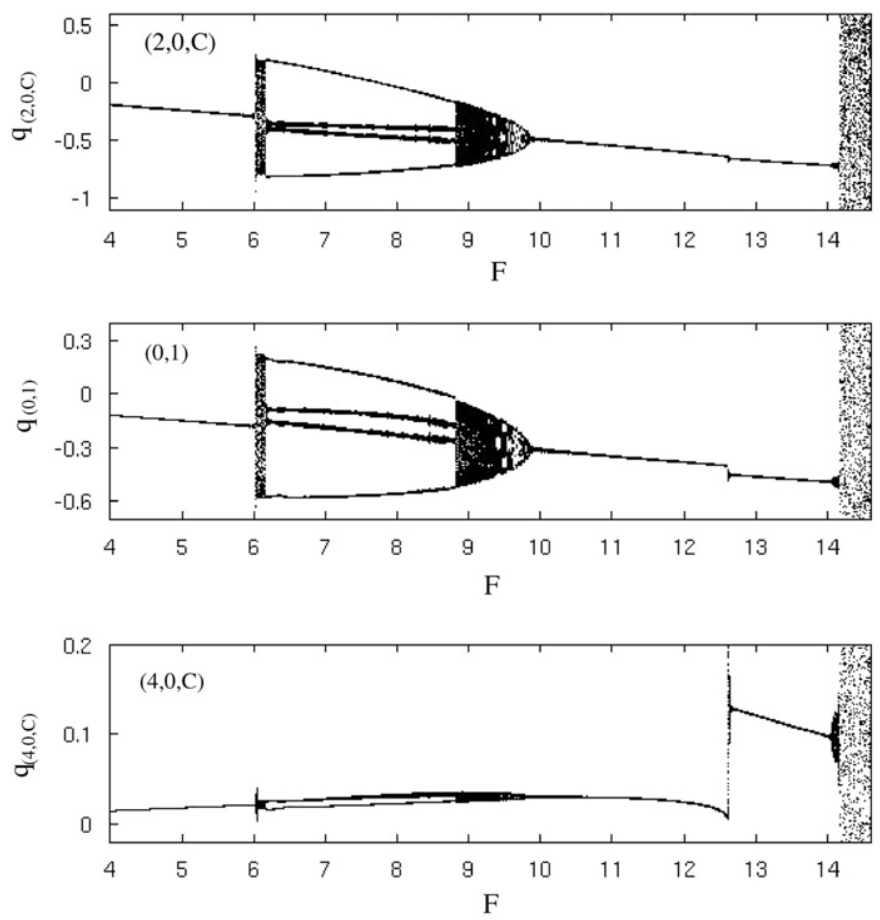

Fig. 6. Bifurcation diagram of Poincaré maps, $\Omega=7.4$ with $F \in[0,15]$. 
the response. From $F=17.4$, a modulated response appears, with strong participation of $(2,0, \mathrm{C})$ and $(0,1)$ that were not excited before. Moreover, we have the relationship:

$2 \omega_{(2,0)}+\omega_{(0,1)}=2 * 5.26+9.06=19.58 \simeq 19.7$,

so that it is very likely that the frequencies of the two modes have been slightly modified by the non-linearity in order to perfectly fulfill the relationship and renders the mode coupling effective. Similarly, a noticeable amplitude is observed on modes $(4,0)$ and $(5,0)$ (not shown), and the relationship:

$\omega_{(5,0)}-\omega_{(4,0)}+\omega_{(0,1)}=20.5$

shows that energy can also be transferred through this relation.

To conclude with these examples, we have shown that although the generic scenario in the very low-frequency range is that of a direct transition to chaotic vibrations, some particular cases exist for limited values of the parameter, so that they are not robust to very slight changes in the parameters. In Section 4 , by adding an imperfection and finely tuning the eigenfrequency, we will show how perfectly matched internal resonance relationships
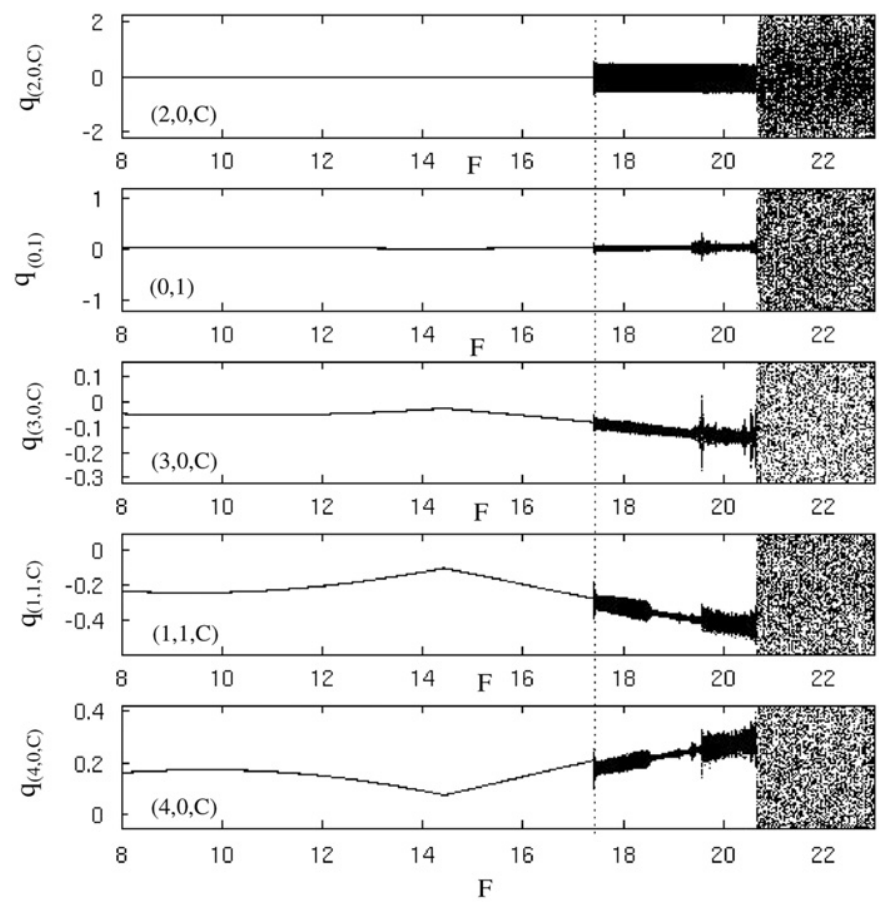

Fig. 7. Bifurcation diagram of Poincaré maps, $\Omega=19.8$ with $F \in[0,23]$.

a

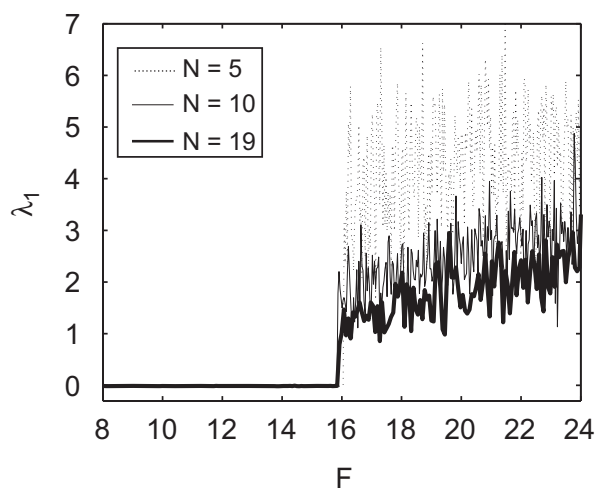

leads to robust and efficient mode coupling and the appearance of coupled solutions with few modes before the chaotic regime.

\subsection{Lyapunov exponents and power spectra}

This section investigates the numerical results obtained in the chaotic regime by inspecting Lyapunov exponents and power spectra of transverse velocity, and reconsiders the modal truncations for the chaotic state. First, the convergence of the maximum Lyapunov exponent is shown in Fig. 8, for $\Omega=5.3$ and 9.4. The maximum Lyapunov exponent $\lambda_{1}$ has been calculated as a sideresult of the computation in the numerical experiments where the forcing amplitude is gradually increased. A positive maximum Laypunov exponent is shown in Fig. 8, evidencing the presence of a chaotic dynamics.

For $\Omega=5.3, \lambda_{1}$ decreases when the number of modes $N$ retained in the truncation increases. Although the difference between $N=10$ and 19 is less significant than between $N=5$ and 10 , the value still continue to slightly decrease, showing that the convergence is more difficult to obtain than for the value of the critical force $F_{c r}$, studied in Section 3.2. For $\Omega=9.4$, the convergence seems to be better but a slight decrease of $\lambda_{1}$ is still observable. This result shows that in the chaotic regime, the convergence is more difficult to obtain and need to be more meticulously studied.

The whole Lyapunov spectrum has been computed for the model with 19 modes. As a second-order problem is at hand, we obtain 38 Lyapunov exponents that are shown in Fig. 9 for $\Omega=5.3$, and two different values of the forcing amplitude: $F=17.1$ and 27.1. The most striking result is the number of positive exponents: half of them are positive, half of them are negative. This indicates, by applying the Lyapunov dimension assumption [53], that the attractor has a dimension equal to that of the phase space. Hence a low-dimensional chaos is not at hand and the trajectories explore the whole phase space. Another striking feature of the Lyapunov spectra, for the two forcings (and for all the cases studied: two other excitation frequencies with 10 level of excitation amplitudes have been computed, yielding the same results), is its symmetry. For Hamiltonian systems, the Lyapunov exponents are symmetric with respect to zero. For assembly of oscillators with the same damping term of the form used here, i.e. $2 \mu \dot{q}_{p}$, see Eq. (8), Dressler showed that the Lyapunov exponent must be symmetric with respect to $-\mu$ [54]. This symmetry property is here exactly verified, i.e. for all $i=1 \ldots N(N=19)$, we have

$\lambda_{i}+\lambda_{N+1-i}=-2 \mu=-0.004$.

b

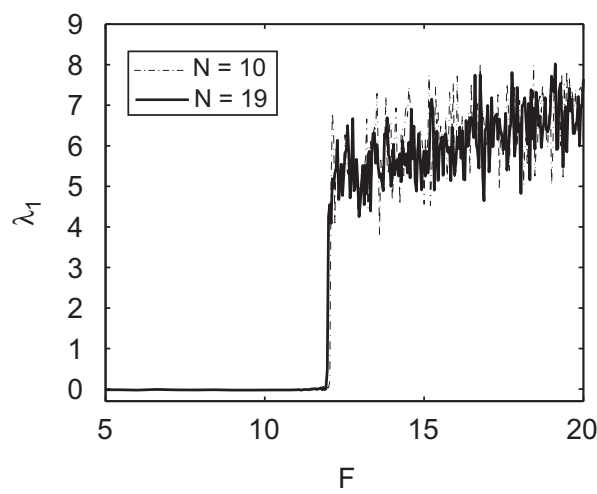

Fig. 8. Convergence of the maximum Lyapunov exponent $\lambda_{1}$ for increasing values of $N$. (a) $\Omega=5$.3. (b) $\Omega=9.4$. 
Dressler also showed that the whole spectrum should verify the following property:

$\sum_{i=1}^{2 N} \lambda_{i}=-2 N \mu$,

which is also perfectly verified by the numerical results.

As a high-dimensional attractor is at hand in the chaotic regime, the framework of wave turbulence is more adapted in order to describe this regime. The numerical experiments reported in previous section and the remarks on Fig. 3 already pointed out that a cascade of energy from the long to the short wavelength was present. Wave (or weak) turbulence describes the distribution of energy for out-of-equilibrium systems, provided that the non-linearity is weak, and that the waves persist (no intermittency), hence allowing one for closed equations that provide analytical predictions for the statistics of cumulants $[29,30]$. The case of plates within the von Kármán assumptions have been tackled in [31], predicting a direct cascade with a power spectrum for the displacement:

$P_{w}(k)=C \frac{P^{1 / 3}}{\left[12\left(1-v^{2}\right)\right]^{1 / 6}} \frac{\ln ^{1 / 3}\left(k_{\star} / k\right)}{\sqrt{E / \rho}} k^{4}$,

where $C$ is a constant, $P$ is the energy flux per unit mass, and $k_{\star}$ an ad-hoc cut-off wave number. The logarithmic correction is weak, so that the main dependence is on $k$ to the power -4 . Translating in the frequency domain via $P_{w}(k) k d k \propto P_{w}(f) d f$ and using the dispersion relation, the prediction for the power spectrum of the

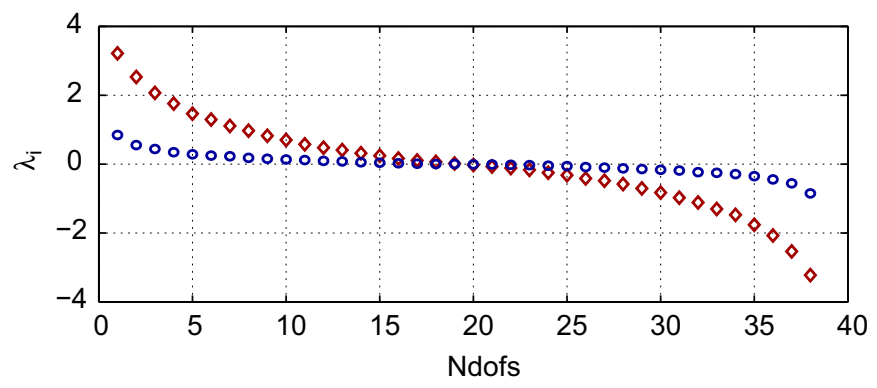

Fig. 9. Lyapunov spectra for $\Omega=5.3, F=17.1(\circ)$ and $F=27.1(\diamond)$.

a

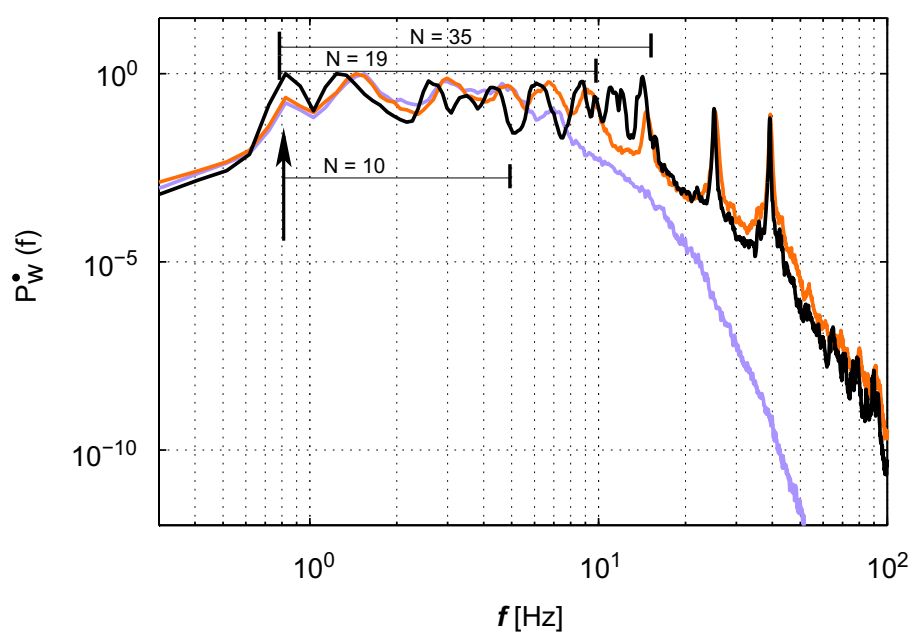

velocity reads $[32,34]$

$$
P_{\dot{w}}(f)=\frac{C^{\prime} P^{1 / 3} h}{\left[12\left(1-v^{2}\right)\right]^{2 / 3}} f^{0},
$$

where $C^{\prime}$ is another constant and the logarithmic correction has not been taken into account. The independence on $f$ has been highlighted by writing explicitly $f^{0}$ in Eq. (18).

Fig. 10 shows $P_{\dot{w}}(f)$ computed by the model, for two different forcing frequencies $\Omega$, and three different truncations: $N=10,19$ and 35 . When increasing the number of modes retained, one can see clearly a first regime which would correspond to the cascade regime, where the power spectrum shows no dependence on $f$ and thus behaves as predicted theoretically by Eq. (18). For $N=10$, which is evidently a too small number of modes for simulating this regime, the cascade range is severely limited, while it is of one decade for $N=35$ and for the two tested excitation frequencies, $\Omega=5.3$ and 20.7. As soon as no more modes are included, the curves change of behaviour and decreases rapidly. Two peaks are visible after the fall, they correspond to the two axisymmetric modes $(0,4)$ and $(0,5)$ that were added in the truncations for stabilization.

To summarize this section, the numerical results clearly show that a limited number of modes are necessary in order to predict the critical value $F_{c r}$ needed for the chaotic vibration to be excited. This is interpreted as a reflection of the fact that the geometry and the stability of the resonance manifold, which is followed in this forced vibration experiment, is completely determined by the slow-flow part and thus by the low-frequency modes. Once the chaotic regime established, numerical results show evidently that a high-dimensional chaos is at hand, in particular by the number of positive Lyapunov exponents. Convergences are then more difficult to attain, which has been evidenced by the convergence of the maximum Lyapunov exponents, as well as the results presented in Fig. 3, where a cascade of energy was highlighted. The more modes we had to the truncation, the more the cascade can broaden its frequency range. Hence this regime has to be interpreted in the framework of wave turbulence (WT), which have been also shown to predict the correct frequency dependence of the velocity power spectrum. Finally, in the WT regime, a very large number of modes (many more than the 35 retained here at maximum) have to be kept until one arrive to the dissipative scale, experimentally evidenced in [32,34], which

b

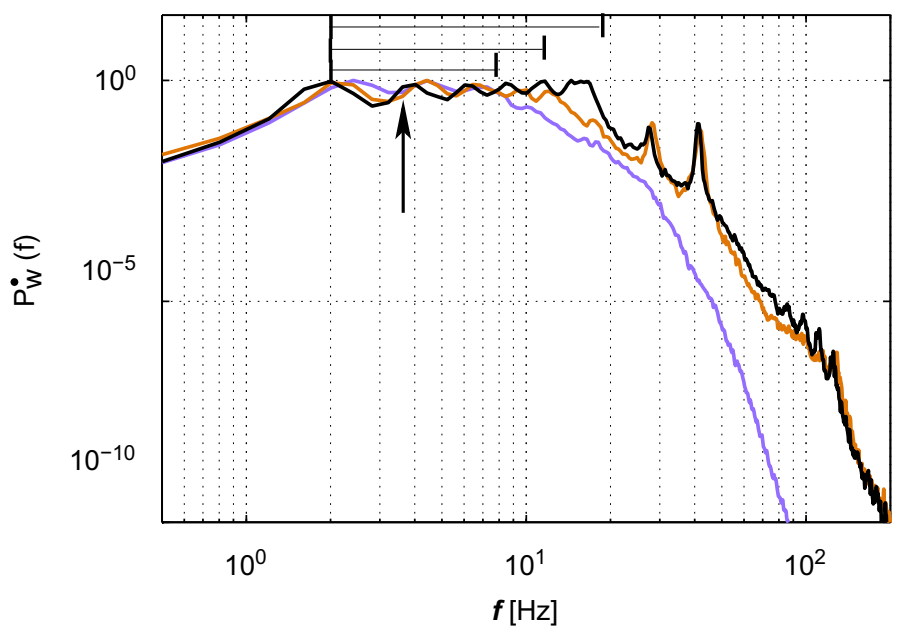

Fig. 10. Power spectra $P_{\dot{w}}(f)$ of the transverse velocity $\dot{w}$, for two different forcing frequencies (indicated by the arrow), and for three different truncations. Left: $\Omega=5.3(f=\Omega / 2 \pi=0.84)$, right: $\Omega=20.7(f=3.29)$. Light gray curve (magenta with colors online): 10 modes truncation $(N=10)$, gray (brown with colors): $N=19$, black: $N=35$. The horizontal segments indicate the frequency band on which the theoretical prediction for $P_{\dot{w}}(f)$ holds (the number of modes $N=10,19$, and 35 are not recalled on the right figure). (For interpretation of the references to color in this figure legend, the reader is referred to the web version of this article.) 
renders the modal method not practically suited for these kind of simulations.

\section{Imperfect plates}

In this section, an imperfect plate having the shape of the first axisymmetric $(0,1)$ mode is considered, see Fig. 11 . For this case, Eq. (6a) reads $w_{0}(r)=a_{(0,1)} \Phi_{(0,1)}(r)$, and the $x$-axis has been chosen so as to cancel the center of mass'offset $z_{g}$. The amplitude of the imperfection is parameterized by $a_{(0,1)}$ only. Evolution of all the linear and non-linear characteristics of this imperfection has already been studied in [37], and the type of non-linearity of the first modes is reported in [55]. Two amplitudes will be studied, $a_{(0,1)}=0.45 h$ ( $h$ is the thickness of the plate), and $a_{(0,1)}=1.86 h$; they have been selected because they create specific internal resonance relationship that will allow for energy transfer and mode coupling before the chaotic state.

\subsection{First imperfection: $a_{(0,1)}=0.45 \mathrm{~h}$}

For this relatively small level of imperfection, the eigenfrequencies are slightly modified as compared to the perfect case, so that the ordering of the modes is left unchanged. This value has been selected so as to raise $\omega_{(0,1)}$ in order to put it exactly at twice the value of $\omega_{(2,0)}$. The first 10 eigenfrequencies are reported in Table 3. One can see that the following internal resonance relationships are fulfilled:

$2 \omega_{(2,0)} \simeq \omega_{(0,1)}$,

$2 \omega_{(0,1)} \simeq \omega_{(1,1)}$

The first numerical result is shown in Fig. 12, and is concerned with the excitation of mode $(2,0, \mathrm{C})$, as $\Omega=5.1$ is chosen, i.e. just below the linear resonance. A 17 modes truncation has been selected, which includes modes $(2,0),(0,1),(3,0),(1,1),(4,0),(5,0)$, $(2,1),(6,0),(0,2)$ and $(0,3)$. The $2: 1$ internal resonance is excited for a very small value of the amplitude of the forcing: $F=0.21$, and leads to a chaotic region where only the first three modes involved in the internal resonance - i.e. $(2,0, C),(2,0, S)$ and $(0,1)-$ participates to the vibration. This regime extends from $F=0.21$ to 1.2 , and is characterized first by a low-dimensional chaos with three modes, then this chaotic regime tends to attain the wave turbulence regime where energy cascades to the high-frequency modes, which is clearly visible by the important values appearing by bursts in $q_{3,0, C}$ and $q_{1,1, C}$ (and visible in all the other coordinates, not shown in the figure) from $F=0.6$. However, the regime is not definitely established and is broken by the appearance of a modulated, quasiperiodic response of the first three modes, for $0.91 \leq F \leq 1.2$. Then the WT regime appears, for $F \geq 1.2$, which is a very small value for the forcing (as compared to the numerical results for the perfect plate) that corresponds to the experimental ones.
A second numerical experiments with this 17 modes truncation is shown in Fig. 13. The forcing frequency is now selected just above the $(0,1)$ eigenfrequency: $\Omega=10.6$. The coupling between the directly excited mode $(0,1)$ and modes $(2,0)$ at half the frequency occurs for a very small value of the forcing $F=0.04$. At the beginning, for $0.04 \leq F \leq 0.65$, the two configurations, $(2,0, C)$ and $(2,0, S)$ are simultaneously excited. But this regime does not appear to be very stable: it is followed by a modulation, and from $F=0.65$, the energy is solely transferred to $(2,0, S)$, which is in agreement with the analytical result obtained in [41] where it was shown that in a 1:1:2 resonance, the energy is transferred to one configuration only. The regime appearing in the response is that of

Table 3

Non-dimensional eigenfrequencies of an imperfect circular plate, imperfection having the shape of mode $(0,1)$ with an amplitude of $a_{(0,1)}=0.45 \mathrm{~h}$.

\begin{tabular}{lllllllllll}
\hline Mode & $(2,0)$ & $(0,1)$ & $(3,0)$ & $(1,1)$ & $(4,0)$ & $(5,0)$ & $(2,1)$ & $(0,2)$ & $(6,0)$ & $(3,1)$ \\
\hline$\omega_{k}$ & 5.26 & 10.52 & 12.24 & 21.13 & 21.53 & 33.06 & 35.51 & 39.00 & 46.81 & 53.05
\end{tabular}
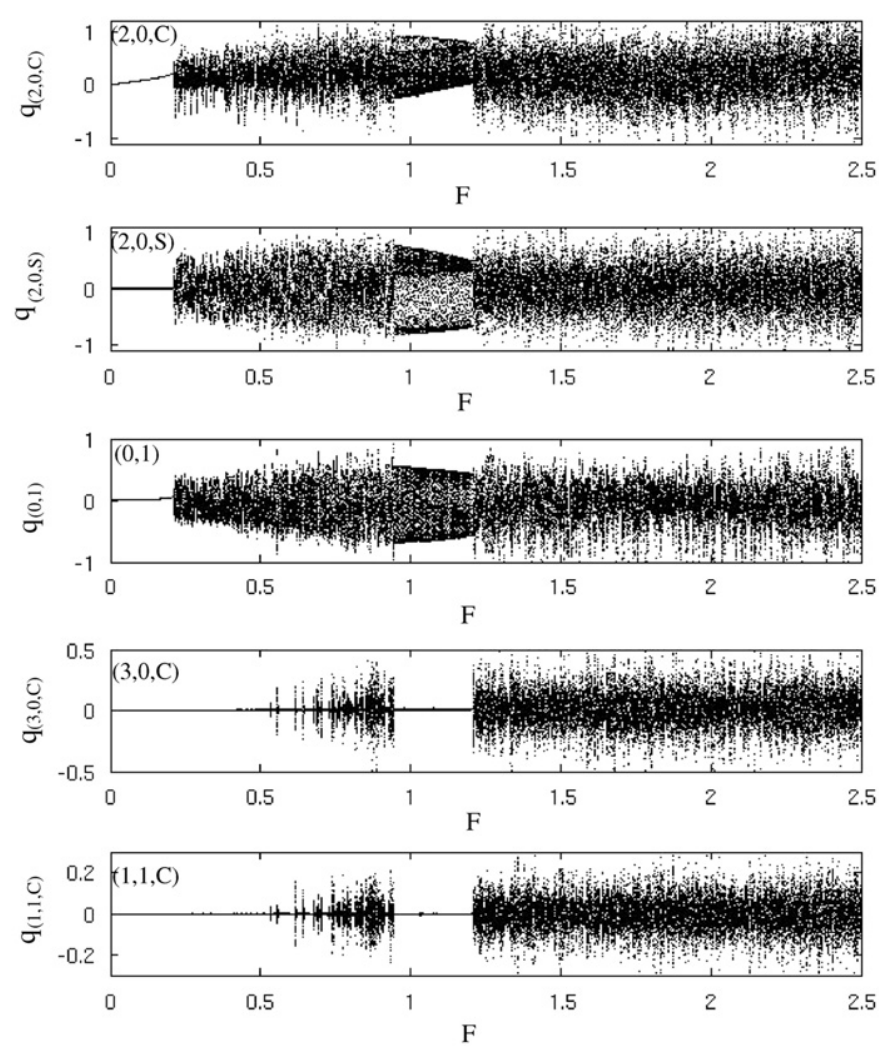

Fig. 12. Bifurcation diagram of Poincaré section for the imperfect plate with $a_{(0,1)}=0.45 h$, excited at $\Omega=5.1$, with an increasing $F$ from 0 to 2.5 . From top to bottom: modes $(2,0, \mathrm{C}),(2,0, \mathrm{~S}),(0,1),(3,0, \mathrm{C})$ and $(1,1, \mathrm{C})$

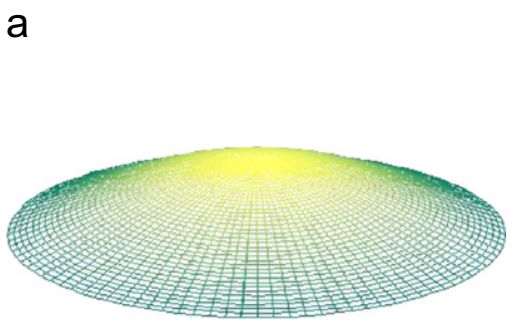

b

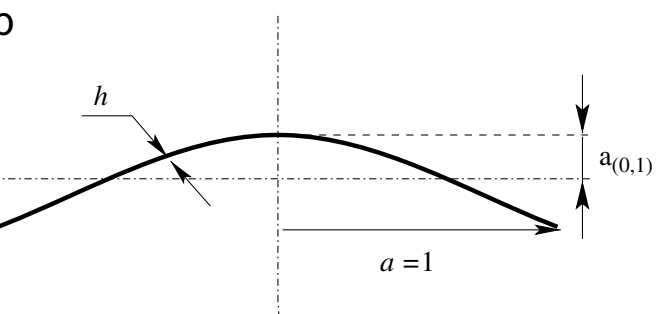

Fig. 11. Imperfect plate having the shape of mode $(0,1)$. (a) 3D view. (b) Cross-section, showing the definition of $a_{(0,1)}$. 
a period-doubling, according to the internal resonance relationship, so that in the Poincaré section two points are visible for each value of the forcing amplitude. For $F=2.25$, the $2: 1$ resonance
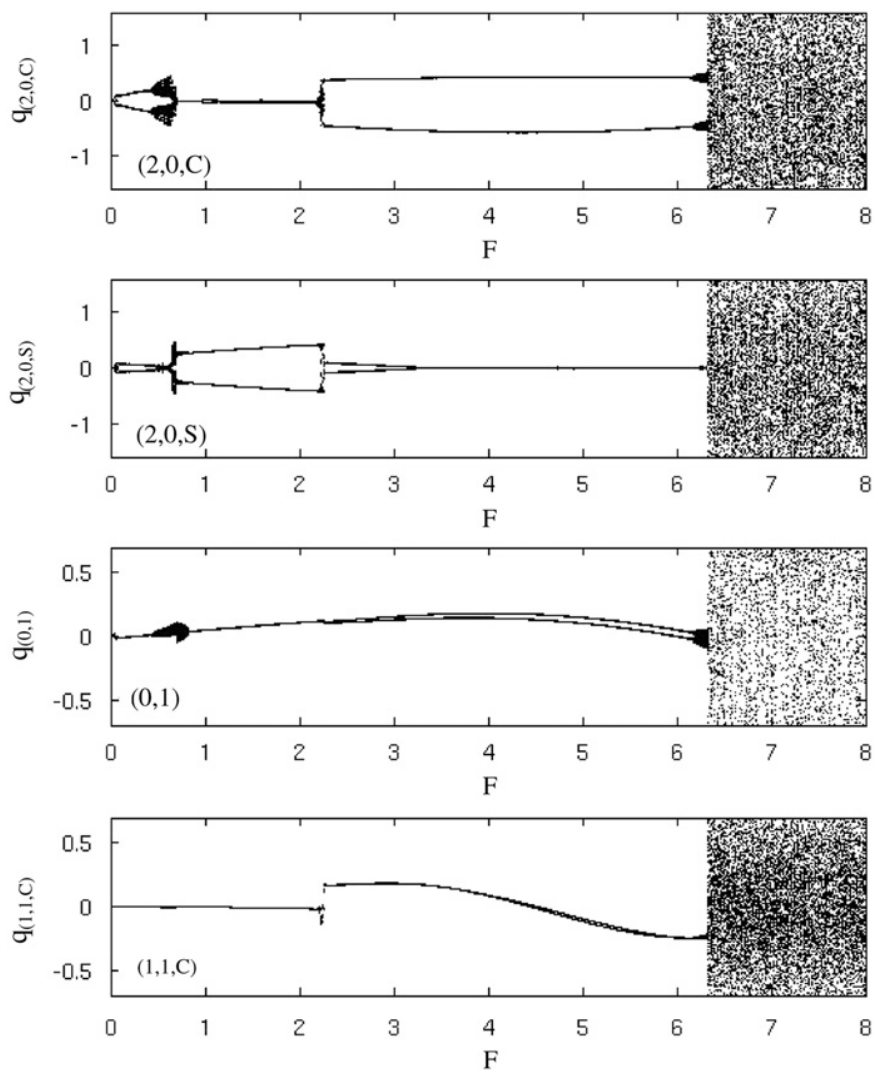

Fig. 13. Bifurcation diagram of Poincaré section for the imperfect plate with $a_{(0,1)}=0.45 h$, excited at $\Omega=10.6$, with an increasing $F$ from 0 to 8 . From top to bottom: modes $(2,0, \mathrm{C}),(2,0, \mathrm{~S}),(0,1)$ and $(1,1, \mathrm{C})$. between $(0,1)$ and $(1,1)$ is suddenly activated, and the amplitude $q_{(1,1, C)}$ jumps from zero to a positive value. This bifurcation also destabilizes the coupling between $(0,1)$ and $(2,0, S)$, and the transfer of energy is then directed to $(2,0, \mathrm{C})$, whereas $q_{(2,0, S)}$ returns to zero. Finally, the chaotic regime sets down for $F=6.3$.

The complete bifurcation diagram $F$ vs $\Omega$ is shown in Fig. 14, for $\Omega \in[1.5,14]$. The $1 / 3$ subharmonics are indicated with a dotted line, whereas the $1 / 2$ subharmonics with dash-dotted lines. The first three eigenfrequencies appearing in the tested frequency range, are indicated. The gray region (green with online colors) represents the chaotic state, while the two light-gray tongues (yellow with online colors) appearing around 5.26 and 10.52 are all the points where the coupling due to the 1:1:2 internal resonance has been numerically observed. In particular, the shape of the coupling $1: 1: 2$ region around mode $(0,1)$ at 10.52 is completely consistent with the theoretical ones that can be found in [41]. For comparison, the limiting value $F_{c r}$ for the perfect plate is reported in Fig. 14 with a dash-dotted line. One can see that adding an imperfection has significantly lowered the forcing levels needed to attain the chaotic regime, so that the amplitudes are now much more consistent with experimental ones. One can conclude that the presence of imperfections, adding quadratic non-linearity and producing second-order internal resonance, is a key factor that makes the chaotic regions more accessible.

\subsection{Second imperfection: $a_{(0,1)}=1.86 \mathrm{~h}$}

To complete the picture provided by this study, the amplitude of the imperfection is raised to $a_{(0,1)}=1.86 h$. The first 10 eigenfrequencies are given in Table 4. This amplitude has been selected as numerous second-order internal resonance relationships appear:

$$
\begin{aligned}
& 2 \omega_{(3,0)} \simeq \omega_{(0,1)}, \\
& 2 \omega_{(0,1)} \simeq \omega_{(0,2)},
\end{aligned}
$$

$\omega_{(3,0)}+\omega_{(5,0)} \simeq \omega_{(6,0)}$.

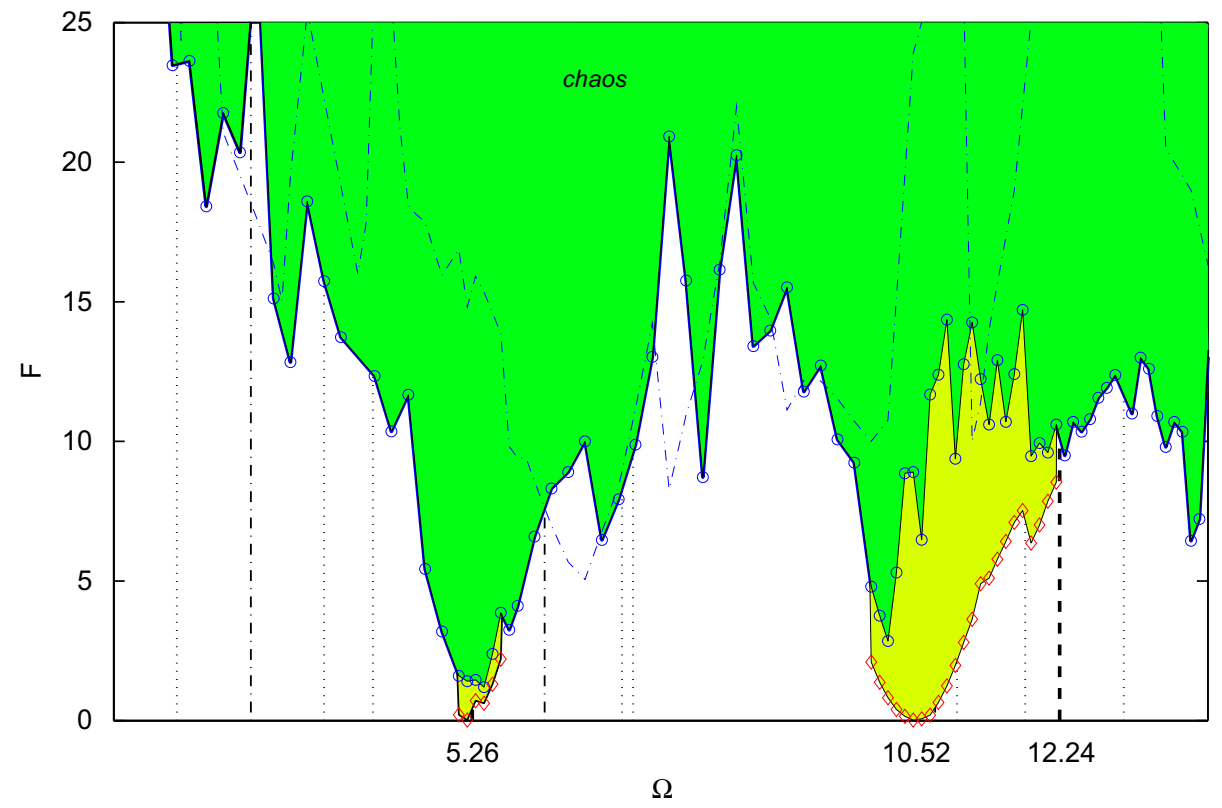

Fig. 14. Complete bifurcation diagram $F$ vs $\Omega$ for the imperfect plate with $a_{(0,1)}=0.45 h$. The gray region (green with online colors) represents the parameter space where chaotic regime is at hand. The two light-gray regions (yellow with online colors) represent the locus where the $1: 1: 2$ resonance is activated. The dash-dotted line recalls the limiting value $F_{c r}$ vs $\Omega$ distinguishing periodic response from chaotic regime for the perfect plate (shown in Fig. 4). (For interpretation of the references to color in this figure legend, the reader is referred to the web version of this article.) 
Moreover, third-order internal resonances are also present in the spectrum, the most striking one being the 1:1 resonance between modes $(0,2)$ and $(6,0)$.

A truncation with 20 modes has been selected, including modes from $(2,0)$ to $(3,1)$ indicated in Table 4 , plus axisymmetric modes $(0,3)$ and $(0,4)$. As in the previous case, the $1: 2$ resonance has been clearly observed by exciting at $\Omega=23.25$ and 46.5 . The case shown in Fig. 15 corresponds to $\Omega=46.8$, i.e. $(6,0, \mathrm{C})$ is the directly excited mode. A first bifurcation occurs at $F=1.8$ where the 1:1 internal resonance becomes activated, so that mode $(0,2)$ gets a non-negligible amplitude. Then for $F=3.5$, a quasiperiodic state with modulation is observed, the two excited modes being $(3,0)$ and $(5,0)$ (the companion configurations $(3,0,5)$ and $(5,0, S)$, not shown in the figure, display the same behaviour). Hence the coupling via the internal resonance shown in Eq. (20c) is activated and energy is transferred to this two modes. Finally the chaotic regime is at hand for $F=8$. This example shows that resonances of the type $1+1: 2$ can also easily be activated, and thus it confirms the scenario exhibited from the experiments, where this kind of couplings was predominantly observed.

\section{Table 4}

Non-dimensional eigenfrequencies of an imperfect circular plate, imperfection having the shape of mode $(0,1)$ with an amplitude of $a_{(0,1)}=1.86 h$.

\begin{tabular}{llllllllllll}
\hline Mode & $(2,0)$ & $(3,0)$ & $(4,0)$ & $(0,1)$ & $(1,1)$ & $(5,0)$ & $(2,1)$ & $(0,2)$ & $(6,0)$ & $(3,1)$ \\
\hline$\omega_{k}$ & 5.28 & 12.27 & 21.56 & 23.25 & 29.21 & 33.09 & 39.55 & 46.50 & 46.84 & 55.07
\end{tabular}
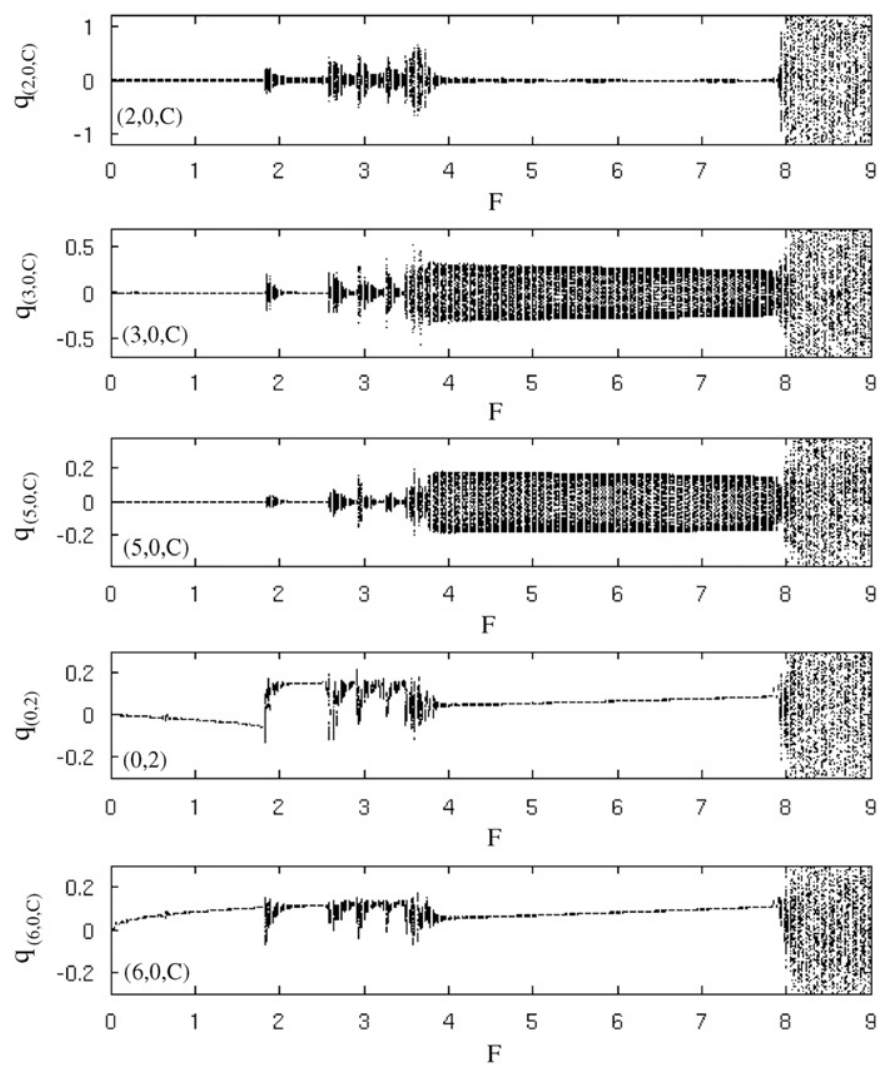

Fig. 15. Bifurcation diagram of Poincare maps for the imperfect plate with $a_{(0,1)}=1.86 h$, excited at $\Omega=46.8$, with an increasing $F$ from 0 to 9 . From top to bottom: modes $(2,0, \mathrm{C}),(3,0, \mathrm{C}),(5,0, \mathrm{C}),(0,2)$ and $(6,0, \mathrm{C})$.

\section{Conclusion}

The transition from periodic to chaotic oscillations has been numerically studied for perfect and imperfect circular plates with a free-edge. The von Kármán PDEs of motion have been discretized with a modal expansion and Galerkin truncations including with up to 35 modes. Consequently, the bifurcation scenario can be studied in the low-frequency part of the structure only, hence limiting the possibilities of internal resonances between the retained modes. Despite this limitation, interesting and convincing results are obtained for the convergence and the dynamical behaviour of forced plates. The three main conclusion of the present study may be summarized as follows:

- The numerical experiment reproduced here, with a slow increase of the forcing amplitude for a constant frequency, may be interpreted from a phase-space point of view, as the search for the stability limit of the forced periodic orbits in the vicinity of the first NNMs. Numerical results clearly pointed out that a small number of modes is enough to determine the critical value $F_{c r}$. Hence the stability limit is completely governed by the slow-flow part of the dynamics.

- The convergence of the solution has been found more difficult to obtain in the chaotic state, highlighting that the framework of wave turbulence is more adapted to describe this regime. The numerical power spectra of the transverse velocity are found to be in agreement with the theoretical prediction of [31], with a cascade regime of increasing bandwidth with increasing $N$. This result raises the following comments:

For the comparison between theory and experiments, it reveals why the convergence results shown on experimental signal in [20,9,7], in terms of dimension estimates, were so difficult to obtain. These studies searched for a lowdimensional process with non-linear signal processing tools [56], whereas a high-dimensional chaos was at hand.

On the other hand, convincing experimental results for a low-dimensional attractor are given in [22]. From the theoretical viewpoint, the investigations by Chueshov and Lasiecka also point out that finite-dimensional attractors and inertial manifolds exist for the von Kármán equations, provided a large amount of damping is considered [26-28]. These two results ask for further numerical investigations for elucidating the transition from low-dimensional chaos to wave turbulence with respect to finite-size effects and the amount of damping.

- Experimental results were summarized in the introduction, underlining the generic route to chaos observed on real plates and shells, with the appearance of a quasiperiodic state characterized by excitation of internally resonant modes. This scenario has been confirmed numerically by finely tuning an imperfection. The energy is then effectively exchanged between the modes before the chaotic regime. Moreover, a global decay of the amplitude of the force needed to attain the WT regime has been clearly evidenced.

\section{Acknowledgments}

The first author (C.T.) would like to warmly thank Olivier Cadot and Arezki Boudaoud for sharing viewpoints on the wave turbulence.

\section{References}

[1] D. Chapelle, K.-J. Bathe, The Finite Element Analysis of Shells-Fundamentals, Springer, Berlin, 2003. 
[2] M. Amabili, Nonlinear Vibrations and Stability of Shells and Plates, Cambridge University Press, 2008.

[3] N.H. Fletcher, Non-linear frequency shifts in quasispherical-cap shells: pitch glide in chinese gongs, Journal of the Acoustical Society of America 78 (6) (1985) 2069-2071.

[4] K. Legge, N.H. Fletcher, Nonlinearity, chaos, and the sound of shallow gongs, Journal of the Acoustical Society of America 86 (6) (1989) 2439-2443.

[5] N.H. Fletcher, T.D. Rossing, The Physics of Musical Instruments, Springer 1998.

[6] S. Bilbao, Numerical Sound Synthesis: Finite Difference Schemes and Simulation in Musical Acoustics, Wiley, 2009.

[7] C. Touzé, A. Chaigne, Lyapunov exponents from experimental time series: application to cymbal vibrations, Acta Acustica 86 (3) (2000) 557-567.

[8] A. Chaigne, C. Touzé, O. Thomas, Nonlinear vibrations and chaos in gongs and cymbals, Acoustical Science and Technology, Acoustical Society of Japan 26 (5) (2005) 403-409.

[9] C. Touzé, A. Chaigne, T. Rossing, S. Schedin, Analysis of cymbal vibrations using non-linear signal processing tools, in: Proceedings of the Internationa Symposium on Musical Acoustics, Leavenworth, USA, 1998, pp. 377-382.

[10] A. Chaigne, C. Touzé, O. Thomas, Non-linear axisymmetric vibrations of gongs, in: Proceedings of the International Symposium on Musical Acoustics, Perugia, Italy, 2001.

[11] C. Touzé, Analyse et modélisation de signaux acoustiques et vibratoires chaotiques. application aux intruments de percussion non-linéaires, Ph.D. Thesis, Université Pierre et Marie Curie, Paris VI, 2000

[12] O. Thomas, Analyse et modélisation de vibrations non-linéaires de milieux minces éla stiques, application aux instruments de percussion, Ph.D. Thesis, Université Pierre et Marie Curie, Paris VI, 2001.

[13] J. Awrejcewicz, V.A. Krysko, A.V. Krysko, Spatio-temporal chaos and solitons exhibited by von Kármán model, International Journal of Bifurcation and Chaos 12 (7) (2002) 1465-1513.

[14] J. Awrejcewicz, V.A. Krysko, G. Narkaitis, Bifurcations of a thin plate-strip excited transversally and axially, Nonlinear Dynamics 32 (2003) 187-209.

[15] J. Awrejcewicz, V.A. Krysko, N. Saveleva, Routes to chaos exhibited by closed flexible cylindrical shells, ASME Journal of Computational and Nonlinear Dynamics 2 (1) (2007) 1-9.

[16] J. Awrejcewicz, V.A. Krysko, I.V. Papkova, Chaotic vibrations of sector-type spherical shells, ASME Journal of Computational and Nonlinear Dynamics 3 (4) (2008) 041005.

[17] M. Amabili, A. Sarkar, M.P. Païdoussis, Chaotic vibrations of circular cylindrical shells: Galerkin versus reduced-order models via the proper orthogonal decomposition method, Journal of Sound and Vibration 290 (3-5) (2006) 736-762.

[18] M. Amabili, Non-linear vibrations of doubly-curved shallow shells, International Journal of Non-linear Mechanics 40 (5) (2005) 683-710.

[19] F. Pellicano, M. Amabili, Dynamic instability and chaos of empty and fluidfilled circular cylindrical shells under periodic axial loads, Journal of Sound and Vibration 293 (1-2) (2006) 227-252.

[20] K.D. Murphy, L.N. Virgin, S.A. Rizzi, Characterizing the dynamic response of a thermally loaded, acoustically excited plate, Journal of Sound and Vibration 196 (5) (1996) 635-658.

[21] K. Nagai, S. Maruyama, M. Oya, T. Yamaguchi, Chaotic oscillations of a shallow cylindrical shell with a concentrated mass under periodic excitation, Computers and Structures 82 (2004) 2607-2619.

[22] K. Nagai, S. Maruyama, T. Murata, T. Yamaguchi, Experiments and analysis on chaotic vibrations of a shallow cylindrical shell-panel, Journal of Sound and Vibration 305 (2007) 492-520.

[23] S. Maruyama, K. Nagai, Y. Tsuruta, Modal interactions in chaotic vibrations of a shallow double-curved shell-panel, Journal of Sound and Vibration 315 (2008) 607-625.

[24] I. Chueshov, Finite dimensionality of an attractor in some problems of nonlinear shell theory, Mathematics of the USSR Sbornik 61 (2) (1998 411-420.

[25] I. Lasiecka, Finite-dimensionality of attractors associated with von Kármán plate equations and boundary damping, Journal of Differential Equations 117 (1995) 357-389

[26] I. Chueshov, I. Lasiecka, Inertial manifolds for von Kármán plate equations Applied Mathematics and Optimization 46 (2002) 179-206.

[27] I. Chueshov, I. Lasiecka, Global attractors for von Kármán evolutions with a nonlinear boundary dissipation, Journal of Differential Equations 198 (2004) 196-231.
[28] I. Chueshov, I. Lasiecka, Attractors and long time behavior of von Kármán thermoelastic plates, Applied Mathematics and Optimization 58 (2008) 195-241.

[29] V.E. Zakharov, V.S. Lvov, G. Falkovisch, Kolmogorov Spectra of Turbulence I: Wave Turbulence, Springer-Verlag, Berlin, 1992.

[30] A. Newell, S. Nazarenko, L. Biven, Wave turbulence and intermittency, Physica D 152-153 (2001) 520-550.

[31] G. Düring, C. Josserand, S. Rica, Weak turbulence for a vibrating plate: can one hear a Kolmogorov spectrum? Physical Review Letters 97 (2006) 025503.

[32] A. Boudaoud, O. Cadot, B. Odille, C. Touzé, Observation of wave turbulence in vibrating plates, Physical Review Letters 100 (2008) 234504.

[33] O. Cadot, A. Boudaoud, C. Touzé, Statistics of power injection in a plate set into chaotic vibration, European Physics Journal B 66 (2008) 399-407.

[34] N. Mordant, Are there waves in elastic wave turbulence? Physical Review Letters 100 (2008) 234505

[35] H.-N. Chu, G. Herrmann, Influence of large amplitudes on free flexural vibrations of rectangular elastic plates, Journal of Applied Mechanics 23 (1956) 532-540

[36] C. Chia, Nonlinear Analysis of Plates, McGraw-Hill, New York, 1980.

[37] C. Camier, C. Touzé, O. Thomas, Non-linear vibrations of imperfect free-edge circular plates and shells, European Journal of Mechanics A/Solids 28 (2009) $500-515$.

[38] G. Ostiguy, S. Sassi, Effects of initial imperfections on dynamic behaviour of rectangular plates, Non-linear Dynamics 3 (1992) 165-181.

[39] M. Amabili, Theory and experiments for large-amplitude vibrations of rectangular plates with geometric imperfections, Journal of Sound and Vibration 291 (3-5) (2006) 539-565.

[40] C. Touzé, O. Thomas, A. Chaigne, Asymmetric non-linear forced vibrations of free-edge circular plates, part I: theory, Journal of Sound and Vibration 258 (4) (2002) 649-676.

[41] O. Thomas, C. Touzé, A. Chaigne, Non-linear vibrations of free-edge thin spherical shells: modal interaction rules and 1:1:2 internal resonance, International Journal of Solids and Structures 42 (11-12) (2005) 3339-3373.

[42] M. Amabili, F. Pellicano, M.P. Païdoussis, Non-linear dynamics and stability of circular cylindrical shells containing flowing fluid, part I: stability, Journal of Sound and Vibration 225 (4) (1999) 655-699.

[43] M. Amabili, F. Pellicano, M.P. Païdoussis, Non-linear dynamics and stability of circular cylindrical shells containing flowing fluid, part III: truncation effect without flow and experiments, Journal of Sound and Vibration 237 (4) (2000) 617-640.

[44] C. Touzé, O. Thomas, Non-linear behaviour of free-edge shallow spherical shells: effect of the geometry, International Journal of Non-linear Mechanics 41 (5) (2006) 678-692.

[45] C.W. Gear, Numerical Initial Value Problems for Ordinary Differential Equations, Prentice-Hall, Englewood Cliffs, 1971.

[46] E. Hairer, G. Wanner, Solving Ordinary Differential Equations II: Stiff and Differential-Algebraic Problems, Springer, 2002.

[47] E. Hairer, C. Lubich, G. Wanner, Geometric Numerical Integration: Structure Preserving Algorithms for Ordinary Differential Equations, Springer, 2006.

[48] L.F. Shampine, M.W. Reichelt, The matlab ode suite, SIAM Journal on Scientific Computing 18 (1) (1997) 1-22.

[49] C. Touzé, O. Thomas, A. Chaigne, Hardening/softening behaviour in non-linear oscillations of structural systems using non-linear normal modes, Journal of Sound and Vibration 273 (1-2) (2004) 77-101.

[50] O. Thomas, C. Touzé, E. Luminais, Non-linear vibrations of free-edge thin spherical shells: experiments on a 1:1:2 internal resonance, Nonlinear Dynamics 49 (1-2) (2007) 259-284.

[51] A.J. Lichtenberg, M.A. Liebermann, Regular and Chaotic Dynamics, Springer, New York, 1992.

[52] A.H. Nayfeh, Nonlinear Interactions: Analytical, Computational and Experimental Methods, Wiley Series in Nonlinear Science, New York, 2000.

[53] J. Farmer, E. Ott, J. Yorke, The dimension of chaotic attractors, Physica D 7 (1-3) (1983) $153-180$.

[54] U. Dressler, Symmetry property of the Lyapunov spectra of a class of dissipative dynamical systems with viscous damping, Physical Review A 38 (4) (1988) 2103-2109.

[55] C. Touzé, C. Camier, G. Favraud, O. Thomas, Effect of imperfections and damping on the type of non-linearity of circular plates and shallow spherical shells, Mathematical Problems in Engineering 2008 (2008) Article ID 678307.

[56] H. Kantz, T. Schreiber, Nonlinear Time Series Analysis, Cambridge University Press, Cambridge, 1997. 